\title{
Compactified Jacobians and $q, t$-Catalan numbers, II
}

\author{
Evgeny Gorsky • Mikhail Mazin
}

Received: 14 September 2012 / Accepted: 4 April 2013 / Published online: 20 April 2013

(C) Springer Science+Business Media New York 2013

\begin{abstract}
We continue the study of the rational-slope generalized $q$, $t$-Catalan numbers $c_{m, n}(q, t)$. We describe generalizations of the bijective constructions of J. Haglund and N. Loehr and use them to prove a weak symmetry property $c_{m, n}(q, 1)=c_{m, n}(1, q)$ for $m=k n \pm 1$. We give a bijective proof of the full symmetry $c_{m, n}(q, t)=c_{m, n}(t, q)$ for $\min (m, n) \leq 3$. As a corollary of these combinatorial constructions, we give a simple formula for the Poincaré polynomials of compactified Jacobians of plane curve singularities $x^{k n \pm 1}=y^{n}$. We also give a geometric interpretation of a relation between rational-slope Catalan numbers and the theory of $(m, n)$-cores discovered by $\mathrm{J}$. Anderson.
\end{abstract}

Keywords $q, t$-Catalan numbers $\cdot$ Compactified Jacobian $\cdot$ Semigroup

\section{Introduction}

Let $m$ and $n$ be two coprime positive integers.

Definition 1 Consider a rectangle with width $m$ and height $n$. The rational-slope Catalan number

$$
c_{m, n}=\frac{(m+n-1) !}{m ! n !}
$$

E. Gorsky

Mathematics Department, Stony Brook University, Stony Brook, NY 11794, USA

e-mail: egorsky@math.sunysb.edu

M. Mazin ( $\varangle)$

Institute for Mathematical Sciences, Stony Brook University, Stony Brook, NY 11794, USA

e-mail: mmazin@math.sunysb.edu 
Fig. 1 Definition of $a(c)$ and $l(c)$

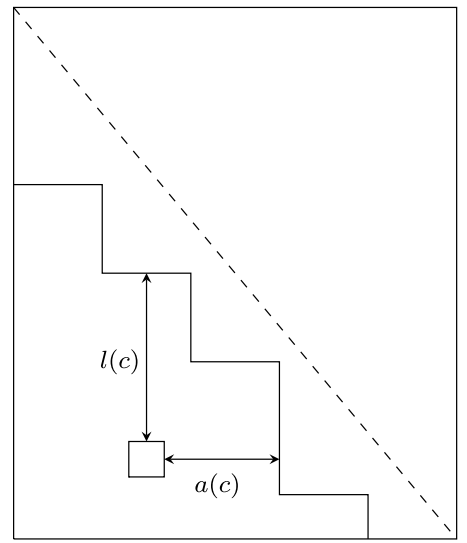

is defined (e.g. [3]) as the number of lattice paths from the northwest corner to the southeast corner of this rectangle, staying below the diagonal connecting these corners. We will denote by $Y_{m, n}$ the set of all such paths, so that $\left|Y_{m, n}\right|=c_{m, n}$.

In the case $m=n+1$ the number $c_{m, n}$ coincides with the usual Catalan number. In [7] M. Haiman and A. Garsia proposed a bivariate generalization of Catalan numbers whose combinatorial definition [8] can be naturally extended to the rationalslope case.

Definition 2 Let $D$ be a Young diagram. Following [18], we define the statistics

$$
h_{+}^{\frac{m}{n}}(D):=\sharp\left\{c \in D: \frac{a(c)}{l(c)+1} \leq \frac{m}{n}<\frac{a(c)+1}{l(c)}\right\},
$$

where $a(c)$ and $l(c)$ denote the arm- and leg-length (see Fig. 1) for a box $c \in D$.

Definition 3 [9] We define the rational-slope $q, t$-Catalan number by the formula

$$
c_{m, n}(q, t)=\sum_{D \in Y_{m, n}} q^{\delta-|D|} t^{h_{+}^{\frac{m}{n}}(D)},
$$

where $\delta=\frac{(m-1)(n-1)}{2}$.

Note that the polynomials $c_{m, n}(q, t)$ are special cases of more general polynomials considered in [18]. For the reader's convenience, from now on we will write $h_{+}$ instead of $h_{+}^{\frac{m}{n}}$.

It has been proved in [8] that $c_{m, n}(q, t)$ coincides with the $q, t$-Catalan numbers of A. Garsia and M. Haiman [7] for $m=n+1$. It has been conjectured in [17] that $c_{n, k n+1}(q, t)$ coincides with the $k$-analogue of them. The following two conjectures are motivated by the above coincidences. Both conjectures appeared previously in the literature, but in different setups. 
Conjecture 1 (Symmetry conjecture) The function $c_{m, n}(q, t)$ satisfies the functional equation

$$
c_{m, n}(q, t)=c_{m, n}(t, q) .
$$

This conjecture is a special case of Conjecture 22 of Loehr and Warrington [18]. The second conjecture is a weaker version of the first one:

Conjecture 2 (Weak symmetry conjecture) The function $c_{m, n}(q, t)$ satisfies the functional equation

$$
c_{m, n}(q, 1)=c_{m, n}(1, q) \text {. }
$$

Conjecture 1 was proved in [8] for $m=n+1$. It was deduced by Garsia and Haglund from some nontrivial identities for $q$, $t$-Catalan numbers. For $m=k n+1$ a similar statement was conjectured in [17]. No bijective proof in any of these cases is known yet.

Theorem 1 The symmetry conjecture holds for $\min (m, n) \leq 3$.

In the proof we construct an explicit bijection exchanging the area and $h_{+}$statistics (see Sect. 4). After the first version of this paper was submitted, K. Lee, L. Li and $\mathrm{N}$. Loehr obtained an independent proof of this result and extended it to the case $m=4$ (see [16]).

The weak symmetry property was proved by an explicit bijective construction for $m=n+1$ by J. Haglund [13] and for $m=k n+1$ by N. Loehr [17]. Following constructions from [9], we introduce a map $G_{m, n}: Y_{m, n} \rightarrow Y_{m, n}$. It was conjectured in [9] that $G_{m, n}$ is a bijection. Let $T: Y_{m, n} \rightarrow Y_{n, m}$ be the transposition map.

Theorem 2 The maps $G_{m, n}$ and $G_{n, m}$ satisfy the following properties:

1. $\left|G_{m, n}(D)\right|=\left|G_{n, m}\left(D^{T}\right)\right|=\delta-h_{+}(D)$.

2. There exists an involution $D \mapsto \widehat{D}$ such that $G_{m, n}(D)=\left(G_{n, m}\left(\widehat{D}^{T}\right)\right)^{T}$.

3. If $m=n+1$, then $Y_{n, n+1}=Y_{n+1, n}$ and, moreover, $G_{n, n+1}=G_{n+1, n}$.

4. If $m=k n \pm 1$, then $G_{m, n}$ is bijective.

5. If $m=k n+1$, then $G_{m, n}$ coincides with the Haglund-Loehr bijection.

Our description of the map $G_{m, n}$ is independent of the Haglund-Loehr construction. We define these maps in terms of semimodules over the semigroup generated by $m$ and $n$. Constructing the inverse of $G_{m, n}$ translates into the reconstruction of a semimodule from a certain collection of data. This approach allowed us to prove bijectivity in the case $m=k n-1$, which is a new result. Using the property (1), one can show that the bijectivity of $G_{m, n}$ (or, equivalently, $G_{n, m}$ ) is sufficient to prove the weak symmetry conjecture.

Corollary 1 The weak symmetry conjecture holds for $m=k n \pm 1$. 
We apply the above combinatorial results to study the geometry of the Jacobi factor, certain moduli spaces associated with plane curve singularities (see [1, 4, 23] and Sect. 2 for the definitions). Of particular interest is the Jacobi factor of the plane curve singularity $\left\{x^{m}=y^{n}\right\}$, which can be identified with a certain affine Springer fiber $[10,14,19]$. It was conjectured in [21] (see also [22]) that the homology of this Jacobi factor is related to the invariants of the $(m, n)$ torus knot. The following theorem is the main result of [9]:

Theorem 3 [9] Consider a plane curve singularity $\left\{x^{m}=y^{n}\right\}$. Then its Jacobi factor admits an affine cell decomposition. The cells are parametrized by Young diagrams $D \in Y_{m, n}$, and the dimension for the cell $C_{D}$ in the Jacobi factor can be computed in terms of $D$ as follows:

$$
\operatorname{dim} C_{D}=\frac{(m-1)(n-1)}{2}-h_{+}(D)
$$

It was pointed out in [9] that the weak symmetry conjecture implies a remarkably simple formula for the Poincaré polynomial of the Jacobi factor:

$$
\begin{aligned}
P_{\overline{J C}_{x}}(t) & =\sum_{D} t^{2 \operatorname{dim} C_{D}}=\sum_{D} t^{2\left(\delta-h_{+}(D)\right)} \\
& =t^{2 \delta} c_{m, n}\left(1, t^{-2}\right) \stackrel{\mathrm{WS}}{=} t^{2 \delta} c_{m, n}\left(t^{-2}, 1\right)=\sum_{D} t^{2|D|} .
\end{aligned}
$$

A similar formula for the Poincaré polynomial was conjectured by Lusztig and Smelt at the last page of [19]. In the case $m=k n \pm 1$ Corollary 1 implies the following result.

Corollary 2 If $m=k n \pm 1$ then the Poincaré polynomial of the Jacobi factor of the plane curve singularity $\left\{x^{m}=y^{n}\right\}$ has the form

$$
P_{\overline{J C}}(t)=\sum_{D} t^{2|D|},
$$

where the summation is done over all Young diagrams $D \in Y_{m, n}$.

Following the ideas of J. Anderson [2], we prove the following result. Recall that $\delta=\frac{(m-1)(n-1)}{2}$. There is a canonical embedding of the Jacobi factor into the Grassmannian $\operatorname{Gr}(\delta, V)$, where $V$ is the space of polynomials in one variable of degree less or equal to $2 \delta-1$. The Grassmannian $\operatorname{Gr}(\delta, V)$ has a cell decomposition by Schubert cells enumerated by Young diagrams contained in a $\delta \times \delta$ square. The cell decomposition of $\overline{J C}_{x}$ is given by intersections with the Schubert cells [11, 23].

Theorem 4 A cell in $\operatorname{Gr}(\delta, V)$ has non-empty intersection with $\overline{J C}_{x}$ if and only if the corresponding Young diagram is a simultaneous $(m, n)$-core, i.e. it does not have hooks of length $m$ or $n$. 


\section{Homology of the compactified Jacobian}

\subsection{Cell decomposition}

In our previous paper [9] we studied the combinatorics of cell decompositions of the Jacobi factors of some plane curve singularities.

Let $x \in C \subset \mathbb{C}^{2}$ be a unibranched plane curve singularity, let $t$ be a normalizing parameter on $C$ at $x$, and $R \subset \mathbb{C}[[t]]$ be the complete local ring at $x$. Let $\delta=$ $\operatorname{dim}(\mathbb{C}[[t]] / R)$. Since $x \in C$ is a plane curve singularity, it follows that $t^{2 \delta} \mathbb{C}[[t]] \subset R$. Let $V=\mathbb{C}[[t]] / t^{2 \delta} \mathbb{C}[[t]]$.

Definition 4 The Jacobi factor $\overline{J C}_{x}$ is the space of $R$-submodules $M \subset \mathbb{C}[[t]]$, such that $M \supset t^{2 \delta} \mathbb{C}[[t]]$ and $\operatorname{dim}(\mathbb{C}[[t]] / M)=\delta$.

In other words, $\overline{J C}_{x}$ is isomorphic to the subvariety of the Grassmannian $\operatorname{Gr}(\delta, V)$, consisting of subspaces invariant under the $R$-action.

Beauville proved in [4] that the compactified Jacobian of a complete rational curve is homeomorphic to the direct product of Jacobi factors of its singularities. To study the topology of the compactified Jacobian of a singular rational curve it is sufficient to study the topology of a single Jacobi factor.

In [23] Piontkowski showed that for some singularities the Jacobi factor admits an algebraic cell decomposition. Following his work, we gave a combinatorial description of the cell decomposition in the case of a plane curve singularity with one Puiseux pair. In particular, it applies to a curve singularity $x^{m}=y^{n}$ for $m$ and $n$ coprime. In this case the Jacobi factor coincides with a certain subvariety of the affine Grassmannian considered first by Lusztig and Smelt in [19] (see also [14]).

Let

$$
\Gamma=\Gamma_{m, n}=\{a m+b n \mid a, b \geq 0\} \subset \mathbb{Z}_{\geq 0}
$$

be the semigroup generated by $m$ and $n$. A subset $\Delta \subset \mathbb{Z}_{\geq 0}$ is called a semimodule over $\Gamma$, if $\Delta+\Gamma \subset \Delta$. It is zero-normalized, if $\min (\Delta)=0$. If not stated otherwise, all semimodules are assumed zero-normalized throughout the paper.

A number $a$ is called an $n$-generator of $\Delta$, if $a \in \Delta$ and $a-n \notin \Delta$. Every $\Gamma$-semimodule has $n$ distinct $n$-generators.

Definition 5 Let $\Delta$ be a $\Gamma$-semimodule, and $x \in \mathbb{Z}$. We define

$$
g_{n, m, \Delta}(x):=\sharp([x, x+m) \backslash \Delta) .
$$

If a semimodule $\Delta$ is fixed, we write $g_{n, m}(x)$ instead of $g_{n, m, \Delta}(x)$ for the reader's convenience.

Theorem 5 [23] The Jacobi factor for a plane curve singularity with one Puiseux pair $(m, n)$ admits an affine cell decomposition. The cells $C_{\Delta}$ are enumerated by the 0-normalized $\Gamma$-semimodules $\Delta$, and their dimensions can be computed by the 
formula

$$
\operatorname{dim} C_{\Delta}=\sum_{j=1}^{n} g_{n, m, \Delta}\left(a_{j}\right)
$$

where $a_{j}$ are the n-generators of $\Delta$.

It has been remarked in [9] that this definition has a natural combinatorial interpretation. Let us label the box $(x, y)$ of the positive quadrant by the number $m n-m(1+x)-n(1+y)$. The corner box $(0,0)$ is labeled by $2 \delta-1=m n-m-n$ and the numbers decrease by $m$ in east direction and by $n$ in north direction. One can check that every number from $\mathbb{N} \backslash \Gamma$ appears exactly once in the $m \times n$ rectangle below the diagonal (it follows e.g. from Lemma 1 below).

Given a $\Gamma$-semimodule $\Delta$, let us mark the boxes labeled by numbers from $\Delta \backslash \Gamma$ and denote the resulting set of boxes by $D(\Delta)$.

Theorem 6 [9] For a $\Gamma$-semimodule $\Delta$ the set $D(\Delta)$ is a Young diagram. The correspondence $D$ between semimodules and $Y_{m, n}$ is bijective. The dimension of a cell $C_{\Delta}$ in the Jacobi factor can be computed in terms of $D(\Delta)$ as follows:

$$
\operatorname{dim} C_{\Delta}=\frac{(m-1)(n-1)}{2}-h_{+}(D(\Delta)) .
$$

Example 1 Consider the semigroup $\Gamma$ generated by the numbers 5 and 7 , and a $\Gamma$-semimodule $\Delta=\mathbb{Z}_{\geq 0} \backslash\{1,2,3,4,6,9\}=\{0,5,7,8,10,11, \ldots\}$. The diagram $D(\Delta)$ is shown in Fig. 2. The 5-generators of $\Delta$ are $\left\{a_{0}, a_{1}, a_{2}, a_{3}, a_{4}\right\}=$ $\{0,7,8,11,14\}$, and

$$
\begin{aligned}
& g_{5,7}(0)=\sharp([0,7) \backslash \Delta)=5, \quad g_{5,7}(7)=\sharp([7,14) \backslash \Delta)=1, \\
& g_{5,7}(8)=\sharp([8,15) \backslash \Delta)=1, \quad g_{5,7}(11)=g_{5,7}(14)=0 .
\end{aligned}
$$

Therefore

$$
\operatorname{dim} C_{\Delta}=g_{5,7}(0)+g_{5,7}(7)+g_{5,7}(8)+g_{5,7}(11)+g_{5,7}(14)=7 .
$$

On the other hand, let us compute $h_{+}(D(\Delta))$. For the corner box (labeled by 23 ) the arm-length equals 1 and the leg-length equals 3 , so the inequality $\frac{5}{7}<\frac{a(c)+1}{l(c)}=\frac{2}{3}$ does not hold. All other boxes of $D(\Delta)$ contribute to $h_{+}$and hence $h_{+}(D(\Delta))=5$, as prescribed by (2).

\subsection{Duality}

Let us recall a useful symmetry property for the plane curve semigroup $\Gamma$.

Lemma 1 (e.g. $[5,15])$ Let $\Gamma$ be the semigroup of a unibranched plane curve singularity and let $\delta$ be its $\delta$-invariant. Then

$$
a \in \Gamma \Longleftrightarrow 2 \delta-1-a \notin \Gamma .
$$


Fig. 2 The diagram $D(\Delta)$ for $m=5, n=7$, and

$\Delta=\mathbb{Z}_{\geq 0} \backslash\{1,2,3,4,6,9\}=$ $\{0,5,7,8,10,11, \ldots\}$

\begin{tabular}{|c|c|c|c|c|}
\hline$\ulcorner 7$ & -14 & -21 & -28 & -35 \\
\hline-2 & -9 & -16 & -23 & -30 \\
\hline 3 & -4 & -11 & -18 & -25 \\
\hline 8 & 1 & $\stackrel{1}{\rightarrow}$ & -13 & -20 \\
\hline 13 & 6 & -1 & -8 & -15 \\
\hline 18 & 11 & 4 & -3 & -10 \\
\hline 23 & 16 & 9 & 2 & -5 \\
\hline & & 14 & 7 & 0 \\
\hline
\end{tabular}

For every $\Gamma$-semimodule $\Delta$ we can consider the dual semimodule

$$
\Delta^{*}:=\{\phi: \phi+\Delta \subset \Gamma\} .
$$

Remark that $\Delta^{*}$ is not zero-normalized.

Lemma 2 The dual semimodule $\Delta^{*}$ can be characterised by the equation

$$
\Delta^{*}=(2 \delta-1)-(\mathbb{Z} \backslash \Delta) .
$$

Proof Note that $(2 \delta-1)-(\mathbb{Z} \backslash \Delta)$ is a $\Gamma$-semimodule. Indeed, if $x \in \mathbb{Z} \backslash \Delta$ then $x-m, x-n \in \mathbb{Z} \backslash \Delta$.

It follows from Lemma 1 that

$$
\phi+\Delta \subset \Gamma \quad \Longleftrightarrow \quad(2 \delta-1) \notin \phi+\Delta .
$$

Indeed, if $(2 \delta-1) \in \phi+\Delta$ then $\phi+\Delta$ is not a subset of $\Gamma$. Conversely, if $\exists x \in$ $(\phi+\Delta) \backslash \Gamma$, then by Lemma $1(2 \delta-1-x) \in \Gamma$, so $(2 \delta-1) \in x+\Gamma \subset \phi+\Delta$. Therefore

$$
\begin{aligned}
\phi+\Delta \subset \Gamma & \Longleftrightarrow(2 \delta-1) \notin \phi+\Delta \\
& \Longleftrightarrow(2 \delta-1-\phi) \notin \Delta \quad \Longleftrightarrow \phi \in(2 \delta-1)-(\mathbb{Z} \backslash \Delta) .
\end{aligned}
$$

Remark 1 Lemmas 2 and 1 are combinatorial analogues of the Gorenstein property for the plane curve singularities (e.g. $[5,15])$.

We normalize $\Delta^{*}$ so that it starts from 0 and denote the normalized semimodule by $\widehat{\Delta}$. Following Lemma 2 , we have

$$
\widehat{\Delta}=\max (\mathbb{Z} \backslash \Delta)-(\mathbb{Z} \backslash \Delta) .
$$


Table 1 Semimodules $\Delta^{*}$ and $\widehat{\Delta}$ for $(m, n)=(3,4)$

\begin{tabular}{lll}
\hline$\Delta$ & $\Delta^{*}$ & $\widehat{\Delta}$ \\
\hline $0,3,4,6, \ldots$ & $0,3,4,6, \ldots$ & $0,3,4,6, \ldots$ \\
$0,3,4,5,6, \ldots$ & $3,4,6, \ldots$ & $0,1,3,4,5,6, \ldots$ \\
$0,2,3,4,5,6, \ldots$ & $4,6, \ldots$ & $0,2,3,4,5,6, \ldots$ \\
$0,1,3,4,5,6, \ldots$ & $3,6, \ldots$ & $0,3,4,5,6, \ldots$ \\
$0,1,2,3,4,5,6, \ldots$ & $6, \ldots$ & $0,1,2,3,4,6, \ldots$ \\
\hline
\end{tabular}

Lemma 3 One has $\widehat{(\widehat{\Delta})}=\Delta$.

Proof It follows from (3) that

$$
\mathbb{Z} \backslash \widehat{\Delta}=\max (\mathbb{Z} \backslash \Delta)-\Delta,
$$

and therefore

$$
\max (\mathbb{Z} \backslash \widehat{\Delta})=\max (\mathbb{Z} \backslash \Delta)
$$

Example 2 We list the combinatorial types of $\Gamma$-semimodules $\Delta, \Delta^{*}$ and $\widehat{\Delta}$ for $(m, n)=(3,4)$ in Table 1 .

\subsection{Maps $G_{m, n}$ and $G_{n, m}$}

Let us return to the formula (1). Given a $\Gamma$-semimodule $\Delta$, we can consider its $m$ generators $a_{1}<\cdots<a_{m}$ and compute

$$
g_{m, n}\left(a_{i}\right)=\sharp\left(\left[a_{i}, a_{i}+n\right) \backslash \Delta\right) .
$$

Theorem 7 [9] The numbers $g_{m, n}\left(a_{i}\right)$ are decreasing. The Young diagram with columns $g_{m, n}\left(a_{i}\right)$ belongs to the set $Y_{m, n}$.

This result allows us to consider the map $G_{m, n}: Y_{m, n} \rightarrow Y_{m, n}$, sending $D(\Delta)$ to the diagram with columns $g_{m, n}\left(a_{i}\right)$. We will also use the notation $G_{m, n}(\Delta)=$ $G_{m, n}(D(\Delta))$ for a $\Gamma$-semimodule $\Delta$. In a similar way, one can define the map $G_{n, m}$ by choosing $n$-generators instead of $m$-generators. Note that the diagram $G_{n, m}(\Delta)$ has $n$ columns, and belongs to $Y_{n, m}$. In this case it is natural to transpose $D(\Delta)$ and consider it as an element of $Y_{n, m}$.

Theorem 8 One has

$$
G_{n, m}(\Delta)=\left(G_{m, n}(\widehat{\Delta})\right)^{T}
$$

Proof Let us recall the notion of an $m$-cogenerator for the $\Gamma$-semimodule $\Delta$ introduced in [9]: we call $b$ an $m$-cogenerator for $\Delta$ if $b \notin \Delta$, but $b+m \in \Delta$. Remark 
that by Lemma 2 the $m$-cogenerators of $\Delta$ are in 1-to- 1 correspondence with the $m$ generators of $\widehat{\Delta}$. More precisely, if $b$ is an $m$-generator of $\widehat{\Delta}$, then $(\max (\mathbb{Z} \backslash \Delta)-b)$ is an $m$-cogenerator for $\Delta$.

Let $a_{1}, \ldots, a_{n}$ be the $n$-generators of $\Delta$ and let $b_{1}, \ldots, b_{m}$ be the $m$-generators of $\widehat{\Delta}$. One can check that $g_{n, m}\left(a_{i}\right)$ is equal to the number of $m$-cogenerators of $\Delta$ greater than $a_{i}$ :

$$
\begin{aligned}
g_{n, m}\left(a_{i}\right) & =\sharp\left(\left[a_{i}, a_{i}+m\right) \backslash \Delta\right)=\sharp\left\{j \mid \max (\mathbb{Z} \backslash \Delta)-b_{j}>a_{i}\right\} \\
& =\sharp\left\{j \mid a_{i}+b_{j}<\max (\mathbb{Z} \backslash \Delta)\right\} .
\end{aligned}
$$

Analogously by Lemma 3

$$
g_{m, n}\left(b_{j}\right)=\sharp\left\{i \mid a_{i}+b_{j}<\max (\mathbb{Z} \backslash \Delta)\right\} .
$$

It is clear that the corresponding Young diagrams are transposed to each other.

Corollary 3 The map $G_{n, m}$ is bijective if and only if the map $G_{m, n}$ is bijective.

Corollary 4 The dimensions of cells in $\overline{J C}_{x}$ labeled by $\Delta$ and $\widehat{\Delta}$ are the same:

$$
\operatorname{dim} C_{\Delta}=\left|G_{n, m}(\Delta)\right|=\left|G_{m, n}(\widehat{\Delta})\right|=\operatorname{dim} C_{\widehat{\Delta}} .
$$

In the case $m=n+1$ the sets $Y_{n, n+1}$ and $Y_{n+1, n}$ coincide. Moreover, we have the following result.

Theorem 9 If $m=n+1$ then $G_{n+1, n}=G_{n, n+1}$.

Proof Let $\Delta$ be a $\Gamma$-semimodule and $x \in \Delta$. Let $a_{+}(x)$ be the minimal $n$-generator of $\Delta$ greater than or equal to $x$. We set $a_{+}(x)=\infty$ if there are no $n$-generators greater than $x$. Similarly, let $a_{-}(x)$ be the maximal $(n+1)$-generator of $\Delta$ less than or equal to $x$. Note that since 0 is an $(n+1)$-generator and $x \in \Delta \subset \mathbb{Z}_{\geq 0}, a_{-}(x)$ is always well defined.

If $x$ is not an $n$-generator then $x-n \in \Delta$, so $(x-n)+(n+1)=x+1 \in \Delta$. If $x+1$ is not an $n$-generator then $x+2 \in \Delta$ etc. By continuing this process we conclude that $\left[x, a_{+}(x)\right] \subset \Delta$. Since $a_{+}(x)-n \notin \Delta$, either $a_{+}(x)+1 \notin \Delta$ or $a_{+}(x)+1$ is a $(n+1)$-generator of $\Delta$.

Analogously, $\left[a_{-}(x), x\right] \subset \Delta$ and either $a_{-}(x)-1 \notin \Delta$ or $a_{-}(x)-1$ is an $n$-generator of $\Delta$. Therefore $n$ - and $(n+1)$-generators of $\Delta$ are split into pairs $\left(a_{-}, a_{+}\right)$such that $\left[a_{-}, a_{+}\right] \subset \Delta$, and this is a 1-to-1 correspondence except for the largest $(n+1)$-generator. Since $\left[a_{-}, a_{+}\right] \subset \Delta$, we have $\left[a_{-}+n, a_{+}+n+1\right] \subset \Delta$ and

$$
g_{n+1, n}\left(a_{-}\right)=\sharp\left(\left[a_{-}, a_{-}+n\right) \backslash \Delta\right)=\sharp\left(\left[a_{+}, a_{+}+n+1\right) \backslash \Delta\right)=g_{n, n+1}\left(a_{+}\right) .
$$

Corollary 5 If $m=n+1$, then

$$
G_{n, n+1}(\widehat{\Delta})=\left(G_{n, n+1}(\Delta)\right)^{T} .
$$


Fig. 3 Maps $G_{m, n}$ and $G_{n, m}$

$G_{3,7}(\cdot)$

$G_{7,3}(\cdot)$

$\Delta$
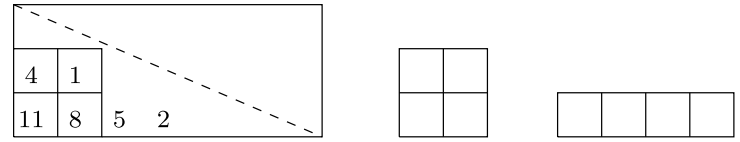

$\widehat{\Delta}$
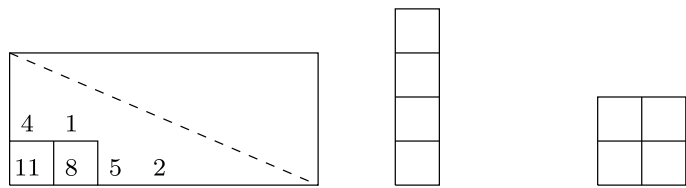

Proof By Theorems 8 and 9

$$
G_{n, n+1}(\widehat{\Delta})=\left(G_{n+1, n}(\Delta)\right)^{T}=\left(G_{n, n+1}(\Delta)\right)^{T} .
$$

Example 3 Let us present an example where $G_{n, m}$ and $G_{m, n}$ are essentially different. Let $(m, n)=(3,7)$ and $\Delta=\{0,1,3,4,6,7,8,9,10,11,12, \ldots\}$. Then the 3 -generators are $0,1,8$, and

$$
g_{3,7}(0)=2, \quad g_{3,7}(1)=2, \quad g_{3,7}(8)=0 .
$$

The 7-generators are $0,1,3,4,6,9,12$, and

$$
g_{7,3}(0)=g_{7,3}(1)=g_{7,3}(3)=g_{7,3}(4)=1, \quad g_{7,3}(6)=g_{7,3}(9)=g_{7,3}(12)=0 .
$$

The dual semimodule is $\widehat{\Delta}=\{0,3,6,7,8, \ldots\}$. Its 3-generators are $0,7,8$, and

$$
g_{3,7}(0)=4, \quad g_{3,7}(7)=g_{3,7}(8)=0
$$

The 7-generators are $0,3,6,8,9,11,12$, and

$$
g_{7,3}(0)=g_{7,3}(3)=2, \quad g_{7,3}(6)=g_{7,3}(8)=g_{7,3}(9)=g_{7,3}(11)=g_{7,3}(12)=0 .
$$

We illustrate this in Fig. 3.

\section{$2.4(m, n)$-Cores}

Definition 6 A Young diagram is called a $p$-core if it does not have boxes with hook length equal to $p$.

The $p$-core partitions play an important role in the study of representations of symmetric groups over finite fields (see [2, 6, 24] and references therein). J. Anderson observed that the number of partitions that are simultaneously $m$ - and $n$-cores is finite: 
Fig. $4(3,4)$ core

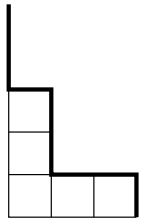

$\begin{array}{llllllll}0 & 1 & 2 & 3 & 4 & 5 & 6 & 7\end{array}$

$\mathrm{N}$ W W N N W N

Theorem 10 [2] The number of partitions that are simultaneously $m$ - and $n$-cores equals

$$
\frac{1}{m+n}\left(\begin{array}{c}
m+n \\
n
\end{array}\right) \text {. }
$$

The proof in [2] uses explicit bijection between the set of $(m, n)$-cores and the set $Y_{m, n}$. Following the analogy between such paths and $\Gamma_{m, n}$-semimodules, we would like to present Anderson's bijection in slightly different form.

Proof Given a set $0 \in \Delta \subset \mathbb{Z}_{\geq 0}$, we construct the partition $P(\Delta)$ by the following rule. We start from $0 \in \Delta$ and read all consecutive integers. If $x \in \Delta$, we move north by 1 , if $x \notin \Delta$, we move west by 1 . The resulting lattice path bounds from above the Young diagram of the partition $P(\Delta)$.

The partition $P(\Delta)$ has a hook of length $m$ if and only if there are integers $x \in \Delta$ and $y \notin \Delta$ such that $y=x+m$. Therefore $P(\Delta)$ is a simultaneous $(m, n)$-core if and only if $\Delta$ is a $\Gamma_{m, n}$-semimodule.

Example 4 The $(3,4)$-core corresponding to $\Delta=\{0,3,4,6, \ldots\}$ is shown in Fig. 4.

Lemma 4 The $(m, n)$-core corresponding to $\widehat{\Delta}$ is conjugate to the $(m, n)$-core for $\Delta$.

Proof By (3), $\widehat{\Delta}=\max (\mathbb{Z} \backslash \Delta)-(\mathbb{Z} \backslash \Delta)$. Therefore to $x \in \Delta$ we associate an $\mathrm{N}$ step in the boundary of the $(m, n)$-core corresponding to $\Delta$ and a $\mathrm{W}$ step in the boundary of the $(m, n)$-core corresponding to $\widehat{\Delta}$. Analogously, to $x \notin \Delta$ we associate a W step in the boundary of the $(m, n)$-core corresponding to $\Delta$ and an $\mathrm{N}$ step in the boundary of the $(m, n)$-core corresponding to $\widehat{\Delta}$.

Theorem 11 The number of self-dual $\Gamma$-semimodules equals

$$
\left(\begin{array}{c}
\left\lfloor\frac{m}{2}\right\rfloor+\left\lfloor\frac{n}{2}\right\rfloor \\
\left\lfloor\frac{m}{2}\right\rfloor
\end{array}\right) .
$$

Proof By Lemma 4 the $\Gamma$-semimodules such that $\Delta=\widehat{\Delta}$ correspond to the selfconjugate $(m, n)$-cores. The number of such cores was computed by B. Ford, H. Mai and L. Sze in [6], and it is equal to $\left(\begin{array}{c}\left\lfloor\frac{m}{2}\right\rfloor+\left\lfloor\frac{n}{2}\right\rfloor \\ \left\lfloor\frac{m}{2}\right\rfloor\end{array}\right)$. 
Remark 2 [3] The number of self-dual modules equals

$$
\left(\begin{array}{c}
\left\lfloor\frac{m}{2}\right\rfloor+\left\lfloor\frac{n}{2}\right\rfloor \\
\left\lfloor\frac{m}{2}\right\rfloor
\end{array}\right)=\left.\frac{[(m+n-1) !]_{q}}{[m !]_{q}[n !]_{q}}\right|_{q=-1} .
$$

Let us give a geometric interpretation of the construction from Theorem 10. As it was discussed in the Sect. 1 , the vector space $V=\mathbb{C}[[t]] / t^{2 \delta} \mathbb{C}[[t]]$ comes with a natural filtration

$$
V=V_{2 \delta} \supset V_{2 \delta-1} \supset \cdots \supset V_{0}=0, \quad V_{i}:=t^{2 \delta-i} V .
$$

Therefore $\operatorname{Gr}(\delta, V)$ has a natural Schubert cell decomposition (see e.g. [20, pp. 74-75]), which can be described as follows.

Let $P$ be a Young diagram contained in a $\delta \times \delta$ square. Let $p_{1} \leq \cdots \leq p_{\delta}$ be its rows. The Schubert cell $C_{P} \subset \operatorname{Gr}(\delta, V)$ consists of subspaces $W \subset V$ such that

$$
\operatorname{dim}\left(W \cap V_{i}\right)=\sharp\left\{j: p_{j}+j \leq i\right\} .
$$

Equivalently, one can assign the diagram $P(W)$ to a subspace $W \subset V$ in the following way. Define the subset $\Delta(W) \subset \mathbb{Z}_{\geq 0}$ as follows:

$$
\Delta(W)=\left\{d \in \mathbb{Z}: \exists p \in W, p \in V_{2 \delta-d} \backslash V_{2 \delta-d-1}\right\} \cup\left[t^{2 \delta}, \infty\right) .
$$

Now let us construct the diagram $P(W)$ from the subset $\Delta(W)$ as in Theorem 10 . One can check that $W$ belongs to the Schubert cell $C_{P(W)} \subset \operatorname{Gr}(\delta, V)$.

Note that according to the construction, $P(W)$ is a simultaneous $(m, n)$-core iff $\Delta(W)$ is a $\Gamma_{m, n}$-semimodule. It follows immediately that if $W \in \overline{J C}_{x}$ then $\Delta(W)$ is a $\Gamma_{m, n}$-semimodule. On the other hand, J. Piontkowski showed in [23] that for a fixed $\Gamma_{m, n}$-semimodule $\Delta$ there always exist $W \in \overline{J C}_{x}$ such that $\Delta(W)=\Delta$. More precisely, modules $W \in \overline{J C}_{x}$ with a fixed semimodule $\Delta(W)$ form a cell in the Piontkowski's cell decomposition of $\overline{J C}_{x}$.

Therefore, one gets the following

Theorem 12 Let $P$ be a Young diagram contained in a $\delta \times \delta$ square. Let $C_{P} \subset$ $\operatorname{Gr}(\delta, V)$ be the corresponding Schubert cell. Then the intersection $C_{P} \cap \overline{J C}_{x}$ is non-empty iff $P$ is a simultaneous $(m, n)$-core, in which case it is the corresponding Piontkowski's affine cell.

\section{Bounce statistics and Poincaré polynomials}

Let $\Delta$ be a $\Gamma_{m, n}$-semimodule. Let us recall the following definition:

Definition 7 A number $a \in \Delta$ is called an $m$-generator if $a-m \notin \Delta$. A number $a \notin \Delta$ is called an $m$-cogenerator if $a+m \in \Delta$. 
In this section we construct the inverse maps $G_{m, n}^{-1}$ in the cases $m=k n \pm 1$. In other words, we reconstruct a $\Gamma_{m, n}$-semimodule $\Delta$ from the collection of integers $g_{m, n}\left(a_{0}\right), g_{m, n}\left(a_{1}\right), \ldots, g_{m, n}\left(a_{m-1}\right)$, where $\left\{0=a_{0}<\cdots<a_{m-1}\right\}$ are the $m$-generators of $\Delta$. Recall that the function $g_{m, n}(x)$ is defined by the formula

$$
g_{m, n}(x)=\sharp([x, x+n) \backslash \Delta) .
$$

For the reader's convenience, we will use $g(x)$ instead of $g_{m, n}(x)$ in this section.

We start with some general facts on generators and cogenerators.

Lemma 5 Suppose that $x \in \Delta$ is not an m-generator. Then $x+n$ is not an m-generator as well.

Proof Indeed, if $x \in \Delta$ is not an $m$-generator, then $x-m \in \Delta$. But then $x-m+n=$ $x+n-m \in \Delta$. Therefore, $x+n$ is not an $m$-generator.

Lemma 6 Fix an integer $x \in \mathbb{Z}$. We have

(a) The number of m-generators in $[x, x+n)$ equals $g(x-m)-g(x)$.

(b) The number of $m$-cogenerators in $[x, x+n)$ equals $g(x)-g(x+m)$.

(c) The number of $m$-generators in the interval $[x, x+n)$ is equal to the number of $n$-cogenerators in the interval $[x-m, x)$.

\section{Proof}

(a) For any integer $y \in[x-m, x-m+n) \backslash \Delta$ the number $y+m$ is either an $m$-generator in $[x, x+n)$ or it is in $[x, x+n) \backslash \Delta$. Also, for any $z \in[x, x+n) \backslash \Delta$ one has $z-m \in[x-m, x-m+n) \backslash \Delta$. Therefore, the number of $m$-generators in $[x, x+m)$ is equal to the number of elements in $[x-m, x-m+n) \backslash \Delta$ minus number of elements in $[x, x+n) \backslash \Delta$, which is $g(x-m)-g(x)$.

(b) Similar to (a): an $m$-cogenerator in $[x, x+n)$ is an integer $y \in[x, x+n) \backslash \Delta$, such that $y+m \notin[x+m, x+m+n) \backslash \Delta$.

(c) Follows immediately from (a) and (b). Indeed, by (a) one gets that the number of $m$-generators in [x, $x+n)$ equals $g(x-m)-g(x)$, and by (b) the number of $n$-cogenerators in $[x-m, x)$ also equals $g(x-m)-g(x)$ (here we switched $m$ and $n$ before applying (b)).

In Sects. 3.1 and 3.3 we discuss the cases $m=k n+1$ and $m=k n-1$, respectively. The logic in both cases is quite similar: first we reconstruct the bounce tree (see Definitions 10 and 13), and then use it to reconstruct the semimodule $\Delta$. In Sect. 3.5 we compare this procedure and the bounce tree with the constructions of Haglund and Loehr.

\subsection{The case $m=k n+1$}

Let $m=k n+1$ from now until the end of the Sect. 3.1. Our goal is to reconstruct the semimodule $\Delta$ from the numbers $g\left(a_{0}\right), \ldots, g\left(a_{k n}\right)$, where $a_{0}, \ldots a_{k n}$ are the $(k n+1)$-generators of $\Delta$ listed in the increasing order. 


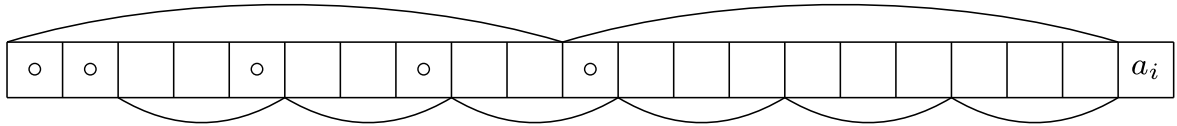

Fig. 5 In this picture $n=3, k=3, a_{i}$ is a 10-generator, and "o" indicates an integer not in $\Delta$. Note that the interval $\left[a_{i}-2(k n+1), a_{i}-2(k n-1)\right]=\left[a_{i}-20, a_{i}-19\right]$ has empty intersection with $\Delta$

Lemma 7 Suppose that $x \in \Delta$ and $x-\alpha n-1 \notin \Delta$ for some $\alpha \in\{0, \ldots, k\}$. Then $x$ is $a(k n+1)$-generator.

Proof Indeed, if $x$ is not a $(k n+1)$-generator, then $x-k n-1 \in \Delta$. But then $(x-k n-1)+(k-\alpha) n=x-\alpha n-1 \in \Delta$. Contradiction.

Definition 8 For $x \in \Delta$ we define $a_{-}(x)$ to be the maximal $(k n+1)$-generator less than or equal to $x$.

Corollary 6 For any $x \in \Delta$ one has $\left[a_{-}(x), x\right] \subset \Delta$.

Proof Follows from Lemma 7 with $\alpha=0$.

Lemma 8 Consider $a(k n+1)$-generator $a_{i}$. Then for any $l>0$, the interval $J_{l}:=$ $\left[a_{i}-l(k n+1), a_{i}-l k n-1\right]$ has empty intersection with $\Delta$.

Proof The proof is by induction on $l$. The case $l=1$ is clear. Then observe that

$$
J_{l+1}=\left\{a_{i}-(l+1)(k n+1)\right\} \cup\left(J_{l}-k n\right) .
$$

We illustrate Lemma 8 with Fig. 5.

Definition 9 For $j>i$ we introduce the following notation:

$$
N_{i j}=\left\lceil\frac{a_{j}-a_{i}}{n}\right\rceil \text { and } K_{i j}=\left\lceil\frac{a_{j}-a_{i}}{k n+1}\right\rceil .
$$

Corollary 7 One has the following formula: $K_{i j}=\left\lceil\frac{N_{i j}}{k}\right\rceil$.

Proof Note that

$$
\left\lceil\frac{N_{i j}}{k}\right\rceil=\left\lceil\frac{\left\lceil\frac{a_{j}-a_{i}}{n}\right\rceil}{k}\right\rceil=\left\lceil\frac{a_{j}-a_{i}}{k n}\right\rceil .
$$

Suppose that $K_{i j} \neq\left\lceil\frac{N_{i j}}{k}\right\rceil$. Then there exists an integer $l$ such that $a_{j}-a_{i} \leq l(k n+1)$ and $a_{j}-a_{i}>l k n$, which is equivalent to $a_{i} \in\left[a_{j}-l(k n+1), a_{j}-l k n\right)$. Therefore by Lemma $8 a_{i} \notin \Delta$. Contradiction.

There are two steps in the reconstruction of $\Delta$. First we reconstruct the bounce tree $T_{\Delta}$, defined below, and then we use it to recover the $(k n+1)$-generators $a_{0}<$ $\cdots<a_{k n}$. 
Fig. 6 The 7-generators of the $\Gamma_{3,7}$-semimodule $\Delta:=\{0,2,3,5,6,7, \ldots\}$ are equal to $0,2,3,5,6,8,11$. The tree $T_{\Delta}$ is presented in the diagram
0

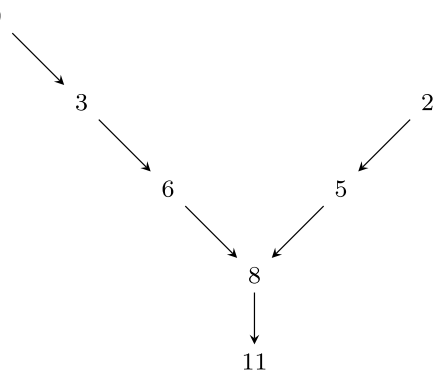

Definition 10 The oriented graph $T_{\Delta}$ is defined as follows. The vertices are the $(k n+1)$-generators of $\Delta: V_{\Delta}=\left\{a_{0}, \ldots, a_{k n}\right\}$. Let $a_{i}, a_{j} \in V_{\Delta}$ be $(k n+1)$ generators. We draw an edge $a_{i} \rightarrow a_{j}$, if $i \neq k n$ and $a_{j}=a_{-}\left(a_{i}+n\right)$. See Fig. 6 for an example of a tree $T_{\Delta}$.

Lemma 9 The graph $T_{\Delta}$ satisfies the following properties:

1. If $a_{i} \rightarrow a_{j}$ is an edge, then $i<j$.

2. The graph $T_{\Delta}$ is a tree with the root $a_{k n}$, and all edges are oriented towards $a_{k n}$.

3. The leaves of $T_{\Delta}$ are the $(k n+1, n)$-generators of $\Delta$, i.e. they are simultaneous $n$ - and $(k n+1)$-generators.

\section{Proof}

1. By construction, $j \geq i$ and $i<k n$. If $i=j$, then there are no $(k n+1)$-generators in the interval $\left[a_{i}+1, a_{i}+n\right]$, hence by Corollary $6\left[a_{i}, a_{i}+n\right] \subset \Delta$ and therefore $\left[a_{i},+\infty\right) \subset \Delta$. By Lemma 5 there are no $(k n+1)$-generators greater than $a_{i}$. Therefore $i=k n$. Contradiction.

2. Follows from the fact that there is only one edge from each vertex except the root $a_{k n}$, and the previous part.

3. If $a_{i}-n \in \Delta$ then by Lemma $5, a_{i}-n$ is a $(k n+1)$-generator. But then the tree $T_{\Delta}$ contains the edge $\left(a_{i}-n\right) \rightarrow a_{i}$ and $a_{i}$ is not a leaf. Therefore, every leaf is a $(k n+1, n)$-generator.

Conversely, suppose that $a_{i}$ is a $(k n+1, n)$-generator, but not a leaf of $T_{\Delta}$. Then there exists a $(k n+1)$-generator $a_{j}$ such that $a_{j} \rightarrow a_{i}$ is an edge of $T_{\Delta}$, so $a_{j}+n>a_{i}$ and the interval $\left[a_{i}+1, a_{j}+n\right]$ does not contain any $(k n+1)$-generators. It then follows from Corollary 6 that the whole interval $\left[a_{i}+1, a_{j}+n\right]$ is contained in $\Delta$. Therefore, by Lemma $7 a_{i}+1-(n+1)=$ $a_{i}-n \in \Delta$. Contradiction.

We will need the following observation about paths in $T_{\Delta}$ :

Lemma 10 Suppose that $a_{i_{0}} \rightarrow a_{i_{1}} \rightarrow \cdots \rightarrow a_{i_{l}}$ is a path in $T_{\Delta}$.

1. The interval $\left[a_{i_{l}}+1, a_{i_{0}}+\ln \right]$ is a subset of $\Delta$ and it does not contain $(k n+1)$ generators. 
2. The number of $(k n+1)$-generators in the interval $\left[a_{i_{0}}+1, a_{i_{0}}+\ln \right]$ is equal to $i_{l}-i_{0}$.

3. If $a_{j}$ is a $(k n+1)$-generator and $a_{i_{l-1}}<a_{j} \leq a_{i_{l}}$, then $N_{i_{0} j}=l$.

Proof The proof of the first part is by induction on $l$. The $l=1$ case is by definition and Corollary 6. Suppose that we proved lemma for $l-1$. Then one has

$$
\left[a_{i_{l}}+1, a_{i_{0}}+\ln \right]=\left[a_{i_{l}}+1, a_{i_{l-1}}+n\right] \cup\left(\left[a_{i_{l-1}}+1, a_{i_{0}}+(l-1) n\right]+n\right) .
$$

The interval $\left[a_{i_{l}}+1, a_{i_{l-1}}+n\right]$ does not contain $(k n+1)$-generators by the definition of the edge $a_{l-1} \rightarrow a_{l}$. In turn, the interval $\left(\left[a_{i_{l-1}}+1, a_{i_{0}}+(l-1) n\right]+n\right)$ does not contain $(k n+1)$-generators by the induction assumption and Lemma 5. Finally, the inclusion $\left[a_{i_{l}}+1, a_{i_{0}}+\ln \right] \subset \Delta$ follows from Corollary 6 .

The second part follows immediately from the first. For the third part, observe that since there are no $(k n+1)$-generators in the interval $\left[a_{i_{l-1}}+1, a_{i_{0}}+(l-1) n\right]$, we get

$$
a_{i_{0}}+(l-1) n<a_{j} \leq a_{i_{l}} \leq a_{i_{0}}+\ln .
$$

Therefore, $N_{i_{0} j}=\left\lceil\frac{a_{j}-a_{i_{0}}}{n}\right\rceil=l$.

Theorem 13 One can reconstruct the tree $T_{\Delta}$ from the collection of numbers $g\left(a_{0}\right), \ldots, g\left(a_{k n}\right)$. More precisely, given these numbers one can list all pairs $(i, j)$ such that $a_{i} \rightarrow a_{j}$ is an edge in $T_{\Delta}$.

Proof We will list the edges of $T_{\Delta}$ in the following order. First, we reconstruct the path from $a_{0}$ to $a_{k n}$. Then we take the smallest $(k n+1)$-generator $a_{l}$, which is not covered yet, and reconstruct the path

$$
a_{l} \rightarrow a_{l+b_{0}^{l}} \rightarrow a_{l+b_{0}^{l}+b_{1}^{l}} \rightarrow \cdots \rightarrow a_{k n}
$$

We repeat this procedure until we run out of generators.

On each step we need to find numbers $b_{0}^{l}, b_{1}^{l}, b_{2}^{l}, \ldots$ By Lemma $10, b_{i}^{l}$ is equal to the number of $(k n+1)$-generators in the interval

$$
I_{i}^{l}:=\left(a_{l}+i n, a_{l}+(i+1) n\right] .
$$

We use this to define $b_{i}^{l}$ for all $i \in \mathbb{Z}$.

We will also need numbers $c_{i}^{l}$, counting $n$-cogenerators in the same intervals $I_{i}^{l}$. According to Lemma $6, b_{i}^{l}$ is equal to the number of $n$-cogenerators in the interval $J_{i}^{l}:=\left(a_{l}+i n-(k n+1), a_{l}+i n\right]$. Note that

$$
J_{i}^{l}=\left\{a_{l}+i n-k n\right\} \sqcup I_{i-k}^{l} \sqcup \cdots \sqcup I_{i-1}^{l} .
$$

By construction, $a_{l}$ is a leaf of the tree $T_{\Delta}$. By Lemma $9, a_{l}$ is a $(k n+1, n)$-generator of $\Delta$. Therefore, $a_{l}+i n-k n=a_{l}+n(i-k)$ is an $n$-cogenerator if and only if 
$i=k-1$. We conclude that $b_{i}^{l}$ can be expressed via $c_{i-k}^{l}, \ldots, c_{i-1}^{l}$ as follows:

$$
b_{i}^{l}= \begin{cases}\sum_{j=i-k}^{i-1} c_{j}^{l} & \text { if } i \neq k-1, \\ 1+\sum_{j=i-k}^{i-1} c_{j}^{l} & \text { if } i=k-1\end{cases}
$$

On the other hand, for $i \geq 0$ one can use Lemmas 6 and 10 to express $c_{i}^{l}$ through $b_{0}^{l}, b_{1}^{l}, \ldots, b_{i}^{l}$ using numbers $g\left(a_{j}\right)$ :

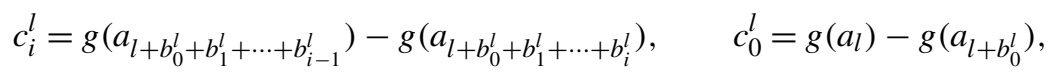

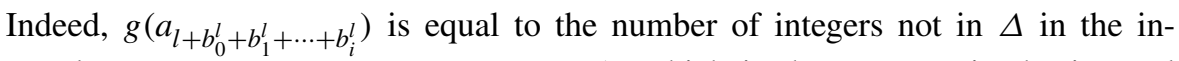

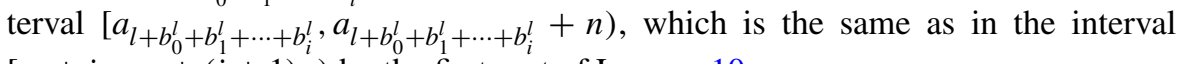
$\left[a_{l}+i n, a_{l}+(i+1) n\right)$ by the first part of Lemma 10 .

Equations (4) and (5) together provide recurrence relations on numbers $b_{i}^{l}$ and $c_{i}^{l}$. To start the recursive algorithm, one needs to compute the initial values $c_{-k}^{l}, \ldots, c_{-1}^{l}$.

Recall the numbers $N_{i j}=\left\lceil\frac{a_{j}-a_{i}}{n}\right\rceil$ and $K_{i j}=\left\lceil\frac{a_{j}-a_{i}}{k n+1}\right\rceil$. Since we know all edges of the tree $T_{\Delta}$ with initial points less than $a_{l}$, we can use Lemma 10 to find numbers $N_{i j}$ for any $i, j \leq l$. Then, using Corollary 7, we can find numbers $K_{i j}$ for any $i, j \leq l$. We can also compute

$$
f\left(a_{i}\right):=\sharp\left(\Delta \cap\left(-\infty, a_{i}\right)\right)=\sum_{j<i} K_{j i},
$$

for all $i \leq l$. Indeed, fix a remainder $0 \leq r<k n+1$, such that the corresponding $(k n+1)$-generator $a_{j} \equiv r(\bmod k n+1)$ is less than $a_{i}$. Then there are exactly $K_{j i}$ integers in $\Delta \cap\left(-\infty, a_{i}\right)$ with remainder $r$ modulo $k n+1$.

Note that

$$
c_{-i}^{l}=\sharp\left(I_{-i+1}^{l} \cap \Delta\right)-\sharp\left(I_{-i}^{l} \cap \Delta\right) \text { for all } i>0 .
$$

Since $a_{l}$ is a $(k n+1, n)$-generator of $\Delta$, we have $a_{l}-i n \notin \Delta$ for $i>0$. Consider the smallest element of $\Delta$ bigger than $a_{l}-i n$. By Corollary 6, it is a $(k n+1)$ generator $a_{\alpha_{i}}$, where $\alpha_{i}:=\min \left\{j: N_{j l} \leq i\right\}$.

Finally, we compute

$$
\begin{aligned}
& \sharp\left(I_{-i}^{l} \cap \Delta\right)=f\left(a_{\alpha_{i-1}}\right)-f\left(a_{\alpha_{i}}\right) \quad \text { for } i>1, \\
& \sharp\left(I_{-1}^{l} \cap \Delta\right)=f\left(a_{l}\right)-f\left(a_{\alpha_{1}}\right)+1,
\end{aligned}
$$

and the number $\sharp\left(I_{0}^{l} \cap \Delta\right)=n-g\left(a_{l}\right)$ is given. Therefore, we can use (6) to find numbers $c_{-k}^{l}, \ldots, c_{-1}^{l}$.

Theorem 14 The tree $T_{\Delta}$ completely determines the semimodule $\Delta$.

Proof Now that we know the whole tree $T_{\Delta}$, we can use Lemma 10 and Corollary 7 to find numbers $K_{i j}$ and $N_{i j}$ for all $i<j \leq k n$. Note that by the definition of num- 
bers $K_{i j}$, we have

$$
a_{i} \in\left(\left(K_{0 i}-1\right)(n k+1), K_{0 i}(n k+1)\right] \text { for all } i .
$$

Therefore, it suffices to recover the remainders $r_{0}, \ldots, r_{k n}$ of the generators $a_{0}, \ldots, a_{k n}$ modulo $k n+1$.

We can use numbers $K_{i j}$ recover the order of $r_{0}, \ldots, r_{k n}$. Indeed, if $i<j$ then

$$
r_{i}<r_{j} \Longleftrightarrow K_{i j}>K_{0 j}-K_{0 i}
$$

Since the remainders $r_{0}, \ldots, r_{k n}$ run through all numbers $0,1, \ldots, k n$ once, knowing the order of $r_{0}, \ldots, r_{k n}$ is equivalent to knowing the remainders themselves.

Let us illustrate Theorems 13 and 14 in the following example.

\subsection{Example: reconstruction of a $\Gamma_{4,9}$-semimodule}

Let $n=4$ and $m=9(k=2)$. Suppose that $g\left(a_{0}\right)=2, g\left(a_{1}\right)=g\left(a_{2}\right)=1$, and $g\left(a_{3}\right)=\cdots=g\left(a_{8}\right)=0$. At the first step we reconstruct the path from $a_{0}=0$ to $a_{8}$ in the tree $T_{\Delta}$. Following the algorithm, we first need to find numbers $c_{-1}^{0}$ and $c_{-2}^{0}$, counting 4-cogenerators in intervals $(-4,0]$ and $(-8,-4]$, respectively. Since there are no elements of $\Delta$ less than $a_{0}=0$, we immediately conclude that

$$
c_{-2}^{0}=\sharp\{\Delta \cap(-4,0]\}-\sharp\{\Delta \cap(-8,-4]\}=1-0=1,
$$

and

$$
c_{-1}^{0}=\sharp\{\Delta \cap(0,4]\}-\sharp\{\Delta \cap(-4,0]\}=\left(4-g\left(a_{0}\right)\right)-1=4-2-1=1 .
$$

Using the recurrence relations (4) and (5) we immediately compute:

$$
\begin{aligned}
& b_{0}^{0}=c_{-2}^{0}+c_{-1}^{0}=2, \quad c_{0}^{0}=g\left(a_{0}\right)-g\left(a_{2}\right)=2-1=1, \\
& b_{1}^{0}=1+c_{-1}^{0}+c_{0}^{0}=3, \quad c_{1}^{0}=g\left(a_{2}\right)-g\left(a_{5}\right)=1, \\
& b_{2}^{0}=c_{0}^{0}+c_{1}^{0}=2, \quad c_{2}^{0}=g\left(a_{5}\right)-g\left(a_{7}\right)=0, \\
& b_{3}^{0}=c_{1}^{0}+c_{2}^{0}=1, \quad c_{3}^{0}=g\left(a_{7}\right)-g\left(a_{8}\right)=0 .
\end{aligned}
$$

Therefore, the path from $a_{0}$ to $a_{8}$ is $a_{0} \rightarrow a_{2} \rightarrow a_{5} \rightarrow a_{7} \rightarrow a_{8}$. The smallest 9 -generator not covered yet is $a_{1}$. Therefore, our next step is to recover the path from $a_{1}$ to $a_{8}$.

Again, we start by reconstructing numbers $c_{-1}^{1}$ and $c_{-2}^{1}$. We have

$$
c_{-2}^{1}=\sharp\left\{\Delta \cap\left(a_{1}-4, a_{1}\right]\right\}-\sharp\left\{\Delta \cap\left(a_{1}-8, a_{1}-4\right]\right\},
$$

and

$$
c_{-1}^{1}=\sharp\left\{\Delta \cap\left(a_{1}, a_{1}+4\right]\right\}-\sharp\left\{\Delta \cap\left(a_{1}-4, a_{1}\right]\right\} .
$$


The only element of $\Delta$ less than $a_{1}$ is $a_{0}$ and, moreover, $a_{0}>a_{1}-4$. Indeed, $a_{0}$ is the only 9-generator less than $a_{1}$ and $a_{1}<a_{2} \leq a_{0}+4$, because we have the arrow $a_{0} \rightarrow a_{2}$ in the tree $T_{\Delta}$. We conclude that

$$
\begin{aligned}
& \sharp\left\{\Delta \cap\left(a_{1}-8, a_{1}-4\right]\right\}=0, \\
& \sharp\left\{\Delta \cap\left(a_{1}-4, a_{1}\right]\right\}=2,
\end{aligned}
$$

and

$$
\sharp\left\{\Delta \cap\left(a_{1}, a_{1}+4\right]\right\}=4-g\left(a_{1}\right)=3 .
$$

Therefore,

$$
c_{-2}^{1}=2 \text { and } \quad c_{-1}^{1}=1 .
$$

We again use the recurrence relations (4) and (5):

$$
\begin{aligned}
& b_{0}^{1}=c_{-2}^{1}+c_{-1}^{1}=3, \quad c_{0}^{1}=g\left(a_{1}\right)-g\left(a_{4}\right)=1, \\
& b_{1}^{1}=1+c_{-1}^{1}+c_{0}^{1}=3, \quad c_{1}^{1}=g\left(a_{4}\right)-g\left(a_{7}\right)=0, \\
& b_{2}^{1}=c_{0}^{1}+c_{1}^{1}=1, \quad c_{2}^{1}=g\left(a_{7}\right)-g\left(a_{8}\right)=0 .
\end{aligned}
$$

Therefore, the path from $a_{1}$ to $a_{8}$ is $a_{1} \rightarrow a_{4} \rightarrow a_{7} \rightarrow a_{8}$. The smallest 9-generator not covered yet is $a_{3}$. Therefore, our next step is to recover the path from $a_{3}$ to $a_{8}$.

Similarly to above, we need to reconstruct numbers $c_{-2}^{3}$ and $c_{-1}^{3}$ first:

$$
c_{-2}^{3}=\sharp\left\{\Delta \cap\left(a_{3}-4, a_{3}\right]\right\}-\sharp\left\{\Delta \cap\left(a_{3}-8, a_{3}-4\right]\right\},
$$

and

$$
c_{-1}^{3}=\sharp\left\{\Delta \cap\left(a_{3}, a_{3}+4\right]\right\}-\sharp\left\{\Delta \cap\left(a_{3}-4, a_{3}\right]\right\} .
$$

There are three 9-generators less than $a_{3}: a_{0}, a_{1}$, and $a_{2}$. Since we have the path $a_{0} \rightarrow$ $a_{2} \rightarrow a_{5}$ in the tree $T_{\Delta}$, we conclude that $a_{0}+8 \geq a_{5}>a_{3}$. Therefore, the only 3 elements of $\Delta$ less than $a_{3}$ are the 9-generators. Furthermore, from the reconstructed part of the tree we see that $a_{3}-8<a_{0}<a_{3}-4, a_{3}-4<a_{1}<a_{3}$, and $a_{3}-4<$ $a_{2}<a_{3}$. Therefore,

$$
\begin{aligned}
& \sharp\left\{\Delta \cap\left(a_{3}-8, a_{3}-4\right]\right\}=1, \\
& \sharp\left\{\Delta \cap\left(a_{3}-4, a_{3}\right]\right\}=3,
\end{aligned}
$$

and

$$
\sharp\left\{\Delta \cap\left(a_{3}, a_{3}+4\right]\right\}=4-g\left(a_{3}\right)=4 .
$$

We conclude that

$$
c_{-2}^{3}=2 \quad \text { and } \quad c_{-1}^{3}=1 .
$$

Once again, we use the recurrence relations (4) and (5):

$$
b_{0}^{3}=c_{-2}^{3}+c_{-1}^{3}=3, \quad c_{0}^{3}=g\left(a_{3}\right)-g\left(a_{6}\right)=0,
$$


Fig. 7 The tree $T_{\Delta}$ for the case $n=4, m=9, g\left(a_{0}\right)=2$, $g\left(a_{1}\right)=g\left(a_{2}\right)=1$, and $g\left(a_{3}\right)=\cdots=g\left(a_{8}\right)=0$ $a_{0}$

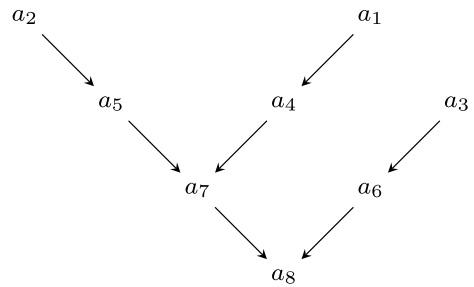

$$
b_{1}^{3}=1+c_{-1}^{3}+c_{0}^{3}=2, \quad c_{1}^{3}=g\left(a_{6}\right)-g\left(a_{8}\right)=0 .
$$

Therefore, the path from $a_{3}$ to $a_{8}$ is $a_{3} \rightarrow a_{6} \rightarrow a_{8}$. See Fig. 7 for the full tree $T_{\Delta}$.

Finally, we reconstruct the semimodule $\Delta$ from the tree $T_{\Delta}$ following the algorithm from Theorem 14. First, we use Lemma 10 to find numbers $N_{0, i}$ for $i=1,2, \ldots, 8$ and Corollary 7 to find $K_{0, i}$. We get

$N_{0,1}=N_{0,2}=1, \quad N_{0,3}=N_{0,4}=N_{0,5}=2, \quad N_{0,6}=N_{0,7}=3, \quad N_{0,8}=4$,

and

$$
K_{0,1}=K_{0,2}=K_{0,3}=K_{0,4}=K_{0,5}=1, \quad K_{0,6}=K_{0,7}=K_{0,8}=2 .
$$

Therefore, we see that $a_{1}, a_{2}, a_{3}, a_{4}$, and $a_{5}$ are in the interval $(0,9)$, while $a_{6}, a_{7}, a_{8}$ are in the interval $(9,18)$.

Now we need to compare the remainders $r_{1}, \ldots, r_{8}$ of the 9-generators $a_{1}, \ldots, a_{8}$. We already know that $r_{1}<r_{2}<r_{3}<r_{4}<r_{5}$ and $r_{6}<r_{7}<r_{8}$. Let us compare $r_{1}$ and $r_{6}$. By Lemma 10 and the tree $T_{\Delta}$, we get $N_{1,6}=2$. Therefore, by Corollary 7 , $K_{1,6}=1$, which means that $a_{6}-a_{1}<9$. Therefore, $r_{6}=a_{6}-9<a_{1}=r_{1}$.

Similarly, one computes that $r_{7}<r_{1}$ and $r_{2}<r_{8}<r_{3}$. Therefore,

$$
r_{6}<r_{7}<r_{1}<r_{2}<r_{8}<r_{3}<r_{4}<r_{5},
$$

or

$$
\begin{array}{llll}
r_{6}=1, & r_{7}=2, & r_{1}=3, & r_{2}=4, \\
r_{8}=5, & r_{3}=6, & r_{4}=7, & r_{5}=8 .
\end{array}
$$

Finally,

$$
\begin{aligned}
& a_{0}=0, \quad a_{1}=r_{1}=3, \quad a_{2}=r_{2}=4, \\
& a_{3}=r_{3}=6, \quad a_{4}=r_{4}=7, \quad a_{5}=r_{5}=8,
\end{aligned}
$$

and

$$
a_{6}=r_{6}+9=10, \quad a_{7}=r_{7}+9=11, \quad a_{8}=r_{8}+9=14 .
$$




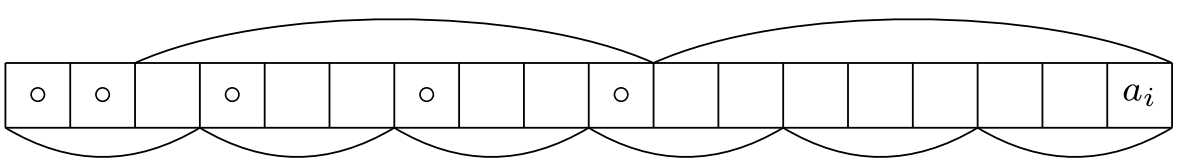

Fig. 8 In this picture $n=3, k=3, a_{i}$ is an 8-generator, and "o" indicates an integer not in $\Delta$. Note that the interval $\left[a_{i}-2 k n+1, a_{i}-2(k n-1)\right]=\left[a_{i}-17, a_{i}-16\right]$ has empty intersection with $\Delta$

\subsection{The case $m=k n-1$.}

Let now $m=k n-1$. In this case the semimodule $\Delta$ can be reconstructed from numbers $g\left(a_{0}\right), \ldots, g\left(a_{k n-2}\right)$ in a way similar to the case $m=k n+1$. However, some adjustments are required. We will omit some of the proofs, in the cases when they are identical to the $m=k n+1$ case.

Lemma 11 Suppose that $x \in \Delta$ and $x-\alpha n+1 \notin \Delta$ for some $\alpha \in\{0, \ldots, k\}$. Then $x$ is a $(k n-1)$-generator.

Proof Indeed, if $x$ is not a $(k n-1)$-generator, then $x-k n+1 \in \Delta$. But then $(x-k n+1)+(k-\alpha) n=x-\alpha n+1 \in \Delta$. Contradiction.

Definition 11 For $x \in \Delta$ we define $a_{+}(x)$ to be the minimal $(k n-1)$-generator greater than or equal to $x$. If there are no $(k n-1)$-generators greater or equal to $x$, we set $a_{+}(x)=\infty$.

Corollary 8 For any $x \in \Delta$ one has $\left[x, a_{+}(x)\right] \subset \Delta$.

Proof Follows from Lemma 11 with $\alpha=0$.

Lemma 12 Consider $a(k n-1)$-generator $a_{i}$. Then for any $l>0$, the interval $J_{l}:=$ $\left[a_{i}-l k n+1, a_{i}-l(k n-1)\right]$ has empty intersection with $\Delta$.

Proof The proof is by induction on $l$. The case $l=1$ is clear. Then observe that

$$
J_{l+1}=\left\{a_{i}-(l+1)(k n-1)\right\} \cup\left(J_{l}-k n\right) .
$$

We illustrate Lemma 12 with Fig. 8.

Definition 12 For $j>i$ we introduce the following notation:

$$
N_{i j}=\left\lfloor\frac{a_{j}-a_{i}}{n}\right\rfloor+1 \text { and } K_{i j}=\left\lfloor\frac{a_{j}-a_{i}}{k n-1}\right\rfloor+1 .
$$

Corollary 9 We have the following formula: $K_{i j}=\left\lceil\frac{N_{i j}}{k}\right\rceil$.

Proof Note that

$$
\left\lceil\frac{N_{i j}}{k}\right\rceil=\left\lceil\frac{\left\lfloor\frac{a_{j}-a_{i}}{n}\right\rfloor+1}{k}\right\rceil=\left\lfloor\frac{a_{j}-a_{i}}{k n}\right\rfloor+1 .
$$


Fig. 9 The 8-generators of the $\Gamma_{3,8}$-semimodule $\Delta:=\{0,3,4,6,7,8, \ldots\}$ are equal to $0,3,4,6,7,9,10,13$. The tree $T_{\Delta}$ is presented in the diagram
0

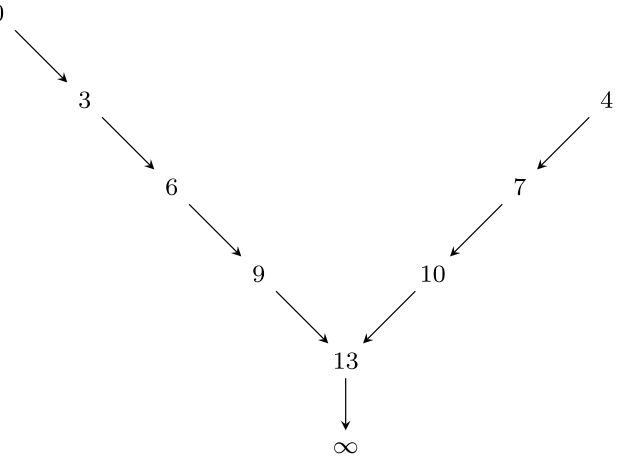

Suppose that $K_{i j} \neq\left\lceil\frac{N_{i j}}{k}\right\rceil$. Then there exists an integer $l$ such that $a_{j}-a_{i} \geq l(k n-1)$ and $a_{j}-a_{i}<l k n$, which is equivalent to $a_{i} \in\left(a_{j}-l k n, a_{j}-l(k n-1)\right]$. Therefore by Lemma $12 a_{i} \notin \Delta$. Contradiction.

Definition 13 The oriented graph $T_{\Delta}$ is defined as follows. The vertices of this graph are the $(k n-1)$-generators of $\Delta$ plus one extra vertex $a_{\infty}: V_{\Delta}=$ $\left\{a_{0}, \ldots, a_{k n-2}, a_{\infty}\right\}$. Let $a_{i}, a_{j} \in V_{\Delta}$ be $(k n-1)$-generators. We draw an edge $a_{i} \rightarrow a_{j}$, if the equation $a_{j}=a_{+}\left(a_{i}+n\right)$ holds. In addition, we draw edges $a_{i} \rightarrow a_{\infty}$ for every $a_{i}$, such that there are no $(k n-1)$-generators greater than or equal to $a_{i}+n$. See Fig. 9 for an example of a tree $T_{\Delta}$.

Lemma 13 The graph $T_{\Delta}$ satisfies the following properties:

1. If $a_{i} \rightarrow a_{j}$ is an edge, then $i<j$.

2. The graph $T_{\Delta}$ is a tree with the root $a_{\infty}$, and all edges are oriented towards $a_{\infty}$.

3. The leaves of $T_{\Delta}$ are the $(k n-1, n)$-generators of $\Delta$.

Proof The first two parts are immediate from the construction. For the third part, consider a $(k n-1)$-generator $a_{i}$. If $a_{i}-n \in \Delta$ then by Lemma $5 a_{i}-n$ is a $(k n-1)$ generator. But then the tree $T_{\Delta}$ contains the edge $\left(a_{i}-n\right) \rightarrow a_{i}$ and $a_{i}$ is not a leaf. Therefore, every leaf is a $(k n-1, n)$-generator.

Conversely, suppose that $a_{i}$ is a $(k n-1, n)$-generator, but not a leaf of $T_{\Delta}$. Then there exists a $(k n-1)$-generator $a_{j}$ such that $a_{j} \rightarrow a_{i}$ is an edge of $T_{\Delta}$, so $a_{j}+n<a_{i}$ and the interval $\left[a_{j}+n, a_{i}-1\right]$ does not contain any $(k n-1)$-generators. It then follows from Corollary 8 that the whole interval $\left[a_{j}+n, a_{i}-1\right]$ is contained in $\Delta$. Therefore by Lemma $11 a_{i}-1-(n-1)=a_{i}-n \in \Delta$. Contradiction.

We will also need the following observation about paths in $T_{\Delta}$ :

Lemma 14 Suppose $a_{i_{0}} \rightarrow a_{i_{1}} \rightarrow \cdots \rightarrow a_{i_{l}}$ is a path in $T_{\Delta}$.

1. The interval $\left[a_{i_{0}}+\ln , a_{i_{l}}-1\right]$ is a subset of $\Delta$ and it does not contain $(k n-1)$ generators. 
2. The number of $(k n-1)$-generators in the interval $\left[a_{i_{0}}, a_{i_{0}}+\ln -1\right]$ is equal to $i_{l}-i_{0}$.

3. If $a_{j}$ is $a(k n-1)$-generator and $a_{i_{l-1}} \leq a_{j}<a_{i_{l}}$, then $N_{i_{0} j}=l$.

Proof The proof of the first part is by induction on $l$. The $l=1$ case is by definition and Corollary 8. Suppose that we proved lemma for $l-1$. Then one has

$$
\left[a_{i_{0}}+\ln , a_{i_{l}}-1\right]=\left(\left[a_{i_{0}}+(l-1) n, a_{i_{l-1}}-1\right]+n\right) \cup\left[a_{i_{l-1}}+n, a_{i_{l}}-1\right] .
$$

The interval $\left[a_{i_{l-1}}+n, a_{i_{l}}-1\right]$ does not contain $(k n-1)$-generators by the definition of the edge $a_{l-1} \rightarrow a_{l}$. In turn, the interval $\left(\left[a_{i_{0}}+(l-1) n, a_{i_{l-1}}-1\right]+n\right)$ does not contain $(k n-1)$-generators by the induction assumption and Lemma 5. Finally, the inclusion $\left[a_{i_{0}}+\ln , a_{i_{l}}-1\right] \subset \Delta$ follows from Corollary 8 .

The second part follows immediately from the first. For the third part, observe that since there are no $(k n-1)$-generators in the interval $\left[a_{i_{0}}+\ln , a_{i_{l}}-1\right]$, we get

$$
a_{i_{0}}+(l-1) n \leq a_{i_{l-1}} \leq a_{j}<a_{i_{0}}+\ln .
$$

Therefore, $N_{i_{0} j}=\left\lfloor\frac{a_{j}-a_{i_{0}}}{n}\right\rfloor+1=l-1+1=l$.

Theorem 15 One can reconstruct the tree $T_{\Delta}$ from the collection of numbers $g\left(a_{0}\right), \ldots, g\left(a_{k n-2}\right)$. More precisely, given these numbers one can list all pairs $(i, j)$ such that $a_{i} \rightarrow a_{j}$ is an edge in $T_{\Delta}$.

Proof We will reconstruct $T_{\Delta}$ in the following order. First, we reconstruct the path from $a_{0}$ to $a_{\infty}$. Then we take the smallest $(k n-1)$-generator $a_{l}$, which is not covered yet, and reconstruct the path

$$
a_{l} \rightarrow a_{l+b_{0}^{l}} \rightarrow a_{l+b_{0}^{l}+b_{1}^{l}} \rightarrow \cdots \rightarrow a_{\infty}
$$

We repeat this procedure until we run out of generators.

On each step all we need to do is to find numbers $b_{0}^{l}, b_{1}^{l}, b_{2}^{l}, \ldots$ According to Lemma $14, b_{i}^{l}$ is equal to the number of $(k n-1)$-generators in the interval $I_{i}^{l}:=$ $\left[a_{l}+i n, a_{l}+(i+1) n\right)$. We use this to define $b_{i}^{l}$ for all $i \in \mathbb{Z}$.

We will also need numbers $c_{i}^{l}$, counting $n$-cogenerators in the same intervals $I_{i}^{l}$. According to Lemma $6, b_{i}^{l}$ is equal to the number of $n$-cogenerators in the interval $J_{i}^{l}:=\left[a_{l}+i n-(k n-1), a_{l}+i n\right)$. Note that

$$
J_{i}^{l}=\left(I_{i-k}^{l} \sqcup \cdots \sqcup I_{i-1}^{l}\right) \backslash\left\{a_{l}+(i-k) n\right\} .
$$

By construction, $a_{l}$ is a leaf of the tree $T_{\Delta}$. By Lemma 13, $a_{l}$ is a $(k n-1, n)$-generator of $\Delta$. Therefore $a_{l}+i n-k n=a_{l}+n(i-k)$ is an $n$-cogenerator if and only if $i=k-1$. We conclude that $b_{i}^{l}$ can be expressed via $c_{i-k}^{l}, \ldots, c_{i-1}^{l}$ as follows:

$$
b_{i}^{l}= \begin{cases}\sum_{j=i-k}^{i-1} c_{j}^{l} & \text { if } i \neq k-1, \\ -1+\sum_{j=i-k}^{i-1} c_{j}^{l} & \text { if } i=k-1 .\end{cases}
$$


On the other hand, for $i \geq 0$ one can use Lemmas 6 and 14 to express $c_{i}^{l}$ through $b_{0}^{l}, b_{1}^{l}, \ldots, b_{i}^{l}$ using numbers $g\left(a_{j}\right)$ :

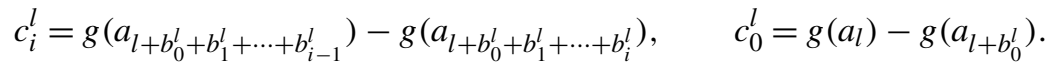

Indeed, $g\left(a_{\left.l+b_{0}^{l}+b_{1}^{l}+\cdots+b_{i}^{l}\right)}\right)$ is equal to the number of integers not in $\Delta$ in the in-

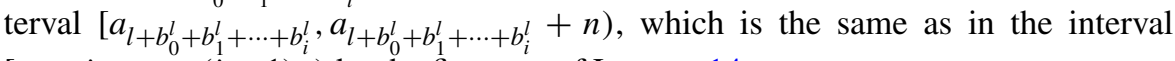
$\left[a_{l}+i n, a_{l}+(i+1) n\right)$ by the first part of Lemma 14 .

Equations (7) and (8) together provide recurrence relations on numbers $b_{i}^{l}$ and $c_{i}^{l}$. To start the recursive algorithm, one needs to find initial values $c_{-k}^{l}, \ldots, c_{-1}^{l}$.

Recall the numbers $N_{i j}=\left\lfloor\frac{a_{j}-a_{i}}{n}\right\rfloor+1$ and $K_{i j}=\left\lfloor\frac{a_{j}-a_{i}}{k n+1}\right\rfloor+1$. Since we know all edges of the tree $T_{\Delta}$ with initial points less than $a_{l}$, we can use Lemma 14 to find numbers $N_{i j}$ for any $i, j \leq l$. Then, by Corollary 9 we can find numbers $K_{i j}$ for any $i, j \leq l$. We can also compute

$$
f\left(a_{i}\right):=\sharp\left(\Delta \cap\left(-\infty, a_{i}\right]\right)=\sum_{j \leq i} K_{j i} .
$$

Indeed, fix a remainder $0 \leq r<k n-1$, such that the corresponding $(k n-1)$ generator $a_{j} \equiv r(\bmod k n-1)$ is less than or equal to $a_{i}$. Then there are exactly $K_{j i}$ integers in $\Delta \cap\left(-\infty, a_{i}\right.$ ] with remainder $r$ modulo $k n-1$.

Note that

$$
c_{-i}^{l}=\sharp\left(I_{-i+1}^{l} \cap \Delta\right)-\sharp\left(I_{-i}^{l} \cap \Delta\right) \text { for all } i>0 .
$$

Since $a_{l}$ is a $(k n-1, n)$-generator of $\Delta$, we have $a_{l}-i n \notin \Delta$ for $i>0$. Consider the biggest element of $\Delta$ less than $a_{l}-i n$. By Corollary 8 , it is a $(k n-1)$-generator $a_{\alpha_{i}}$, where $\alpha_{i}:=\max \left\{j \mid N_{j l}>i\right\}$. If the set $\left\{j \mid N_{j l}>i\right\}$ is empty, then $a_{l}-i n \leq 0$ and there are no elements of $\Delta$ less than $a_{l}-i n$. For consistency, we set $\alpha_{i}=-1$, $a_{-1}=-\infty$, and $f(-\infty)=0$ in this case.

Finally, we compute

$$
\begin{aligned}
& \sharp\left(I_{-i}^{l} \cap \Delta\right)=f\left(a_{\alpha_{i-1}}\right)-f\left(a_{\alpha_{i}}\right) \quad \text { for } i>1, \\
& \sharp\left(I_{-1}^{l} \cap \Delta\right)=f\left(a_{l}\right)-f\left(a_{\alpha_{1}}\right)-1,
\end{aligned}
$$

and the number $\sharp\left(I_{0}^{l} \cap \Delta\right)=n-g\left(a_{l}\right)$ is given. Therefore, we can use (9) to find numbers $c_{-k}^{l}, \ldots, c_{-1}^{l}$.

Theorem 16 The tree $T_{\Delta}$ completely determines the semimodule $\Delta$.

Proof The same as in the $m=k n+1$ case.

3.4 Example: reconstruction of a $\Gamma_{5,9}$-semimodule

Let $n=5, k=2$, and $m=2 \times 5-1=9$. Suppose that $g\left(a_{0}\right)=3, g\left(a_{1}\right)=g\left(a_{2}\right)=$ $g\left(a_{3}\right)=2, g\left(a_{4}\right)=1$, and $g\left(a_{5}\right)=\cdots=g\left(a_{8}\right)=0$. At the first step we reconstruct the path from $a_{0}=0$ to $a_{\infty}$ in the tree $T_{\Delta}$. Following the algorithm, we first 
need to find numbers $c_{-1}^{0}$ and $c_{-2}^{0}$, counting 5-cogenerators in intervals $[-5,0)$ and $[-10,-5)$, respectively. Since there are no elements of $\Delta$ less than $a_{0}=0$, we immediately conclude that

$$
c_{-2}^{0}=\sharp\{\Delta \cap[-5,0)\}-\sharp\{\Delta \cap[-10,-5)\}=0-0=0,
$$

and

$$
c_{-1}^{0}=\sharp\{\Delta \cap[0,5)\}-\sharp\{\Delta \cap[-5,0)\}=\left(5-g\left(a_{0}\right)\right)-0=2 .
$$

Using the recurrence relations (7) and (8) we immediately compute:

$$
\begin{aligned}
& b_{0}^{0}=c_{-2}^{0}+c_{-1}^{0}=2, \quad c_{0}^{0}=g\left(a_{0}\right)-g\left(a_{2}\right)=3-2=1, \\
& b_{1}^{0}=-1+c_{-1}^{0}+c_{0}^{0}=2, \quad c_{1}^{0}=g\left(a_{2}\right)-g\left(a_{4}\right)=2-1=1, \\
& b_{2}^{0}=c_{0}^{0}+c_{1}^{0}=2, \quad c_{2}^{0}=g\left(a_{4}\right)-g\left(a_{6}\right)=1, \\
& b_{3}^{0}=c_{1}^{0}+c_{2}^{0}=2 .
\end{aligned}
$$

Therefore, the path from $a_{0}$ to $a_{\infty}$ is $a_{0} \rightarrow a_{2} \rightarrow a_{4} \rightarrow a_{6} \rightarrow a_{8} \rightarrow a_{\infty}$. The smallest 9 -generator not covered yet is $a_{1}$. Therefore, our next step is to recover the path from $a_{1}$ to $a_{\infty}$.

Again, we start by reconstructing numbers $c_{-1}^{1}$ and $c_{-2}^{1}$. We have

$$
c_{-2}^{1}=\sharp\left\{\Delta \cap\left[a_{1}-5, a_{1}\right)\right\}-\sharp\left\{\Delta \cap\left[a_{1}-10, a_{1}-5\right)\right\},
$$

and

$$
c_{-1}^{1}=\sharp\left\{\Delta \cap\left[a_{1}, a_{1}+5\right)\right\}-\sharp\left\{\Delta \cap\left[a_{1}-5, a_{1}\right)\right\} .
$$

The only 9-generator of $\Delta$ less than $a_{1}$ is $a_{0}$ and, moreover, $a_{0}>a_{1}-5$. In fact, $a_{0}+5=a_{2}>a_{1}$. Indeed, $a_{0}+5=5$ is a 9-generator, and we have the arrow $a_{0} \rightarrow a_{2}$ in the tree $T_{\Delta}$. We conclude that

$$
\begin{aligned}
& \sharp\left\{\Delta \cap\left[a_{1}-10, a_{1}-5\right)\right\}=0, \\
& \sharp\left\{\Delta \cap\left[a_{1}-5, a_{1}\right)\right\}=1,
\end{aligned}
$$

and

$$
\sharp\left\{\Delta \cap\left[a_{1}, a_{1}+5\right)\right\}=5-g\left(a_{1}\right)=3 .
$$

Therefore,

$$
c_{-2}^{1}=1 \quad \text { and } \quad c_{-1}^{1}=2 .
$$

We again use the recurrence relations (7) and (8):

$$
\begin{aligned}
& b_{0}^{1}=c_{-2}^{1}+c_{-1}^{1}=3, \quad c_{0}^{1}=g\left(a_{1}\right)-g\left(a_{4}\right)=2-1=1, \\
& b_{1}^{1}=-1+c_{-1}^{1}+c_{0}^{1}=2, \quad c_{1}^{1}=g\left(a_{4}\right)-g\left(a_{6}\right)=1, \\
& b_{2}^{1}=c_{0}^{1}+c_{1}^{1}=2, \quad c_{2}^{1}=g\left(a_{6}\right)-g\left(a_{8}\right)=0 .
\end{aligned}
$$


Therefore, the path from $a_{1}$ to $a_{\infty}$ is $a_{1} \rightarrow a_{4} \rightarrow a_{6} \rightarrow a_{8} \rightarrow a_{\infty}$. The smallest 9-generator not covered yet is $a_{3}$. Therefore, our next step is to recover the path from $a_{3}$ to $a_{\infty}$.

Similarly to the above, we need to reconstruct numbers $c_{-2}^{3}$ and $c_{-1}^{3}$ first:

$$
c_{-2}^{3}=\sharp\left\{\Delta \cap\left[a_{3}-5, a_{3}\right)\right\}-\sharp\left\{\Delta \cap\left[a_{3}-10, a_{3}-5\right)\right\},
$$

and

$$
c_{-1}^{3}=\sharp\left\{\Delta \cap\left[a_{3}, a_{3}+5\right)\right\}-\sharp\left\{\Delta \cap\left[a_{3}-5, a_{3}\right)\right\} .
$$

There are three 9-generators less than $a_{3}: a_{0}, a_{1}$, and $a_{2}$. Since we already know the path $a_{0} \rightarrow a_{2} \rightarrow a_{4}$, we immediately conclude that $a_{3}<a_{0}+10$. Since $a_{3}$ and $a_{0}$ have different remainders modulo 9 , we get $a_{3}<a_{0}+9$. Therefore, there are no elements of $\Delta$ less than or equal to $a_{3}-9$. We conclude that $a_{0}, a_{1}$, and $a_{2}$ are the only elements of $\Delta$ less than $a_{3}$. Moreover, $a_{2}+5>a_{3}$ and $a_{1}+5>a_{3}$, while $a_{0}+5=a_{2}<a_{3}<a_{0}+10$. Therefore,

$$
\begin{aligned}
& \sharp\left\{\Delta \cap\left[a_{3}-10, a_{3}-5\right)\right\}=1, \\
& \sharp\left\{\Delta \cap\left[a_{3}-5, a_{3}\right)\right\}=2,
\end{aligned}
$$

and

$$
\sharp\left\{\Delta \cap\left[a_{3}, a_{3}+5\right)\right\}=5-g\left(a_{3}\right)=3 .
$$

We conclude that

$$
c_{-2}^{3}=1 \quad \text { and } \quad c_{-1}^{3}=1 .
$$

Once again, we use the recurrence relations (7) and (8):

$$
\begin{aligned}
& b_{0}^{3}=c_{-2}^{3}+c_{-1}^{3}=2, \quad c_{0}^{3}=g\left(a_{3}\right)-g\left(a_{5}\right)=2, \\
& b_{1}^{3}=-1+c_{-1}^{3}+c_{0}^{3}=2, \quad c_{1}^{3}=g\left(a_{5}\right)-g\left(a_{7}\right)=0 . \\
& b_{2}^{3}=c_{0}^{3}+c_{1}^{3}=2 .
\end{aligned}
$$

Therefore, the path from $a_{3}$ to $a_{\infty}$ is $a_{3} \rightarrow a_{5} \rightarrow a_{7} \rightarrow a_{\infty}$. See Fig. 10 for the full tree $T_{\Delta}$.

Finally, we reconstruct the semimodule $\Delta$ from the tree $T_{\Delta}$ following the algorithm from Theorem 14. First, we use Lemma 14 to find numbers $N_{0, i}$ for $i=1,2, \ldots, 8$ and Corollary 9 to find $K_{0, i}$. We get

$$
\begin{aligned}
& N_{0,1}=1, \quad N_{0,2}=N_{0,3}=2, \quad N_{0,4}=N_{0,5}=3, \\
& N_{0,6}=N_{0,7}=4, \quad N_{0,8}=5,
\end{aligned}
$$

and

$$
K_{0,1}=K_{0,2}=K_{0,3}=1, \quad K_{0,4}=K_{0,5}=K_{0,6}=K_{0,7}=2, \quad K_{0,8}=3 .
$$


Fig. 10 The tree $T_{\Delta}$ for the case $n=5, m=9, g\left(a_{0}\right)=3$, $g\left(a_{1}\right)=g\left(a_{2}\right)=g\left(a_{3}\right)=2$, $g\left(a_{4}\right)=1$, and $g\left(a_{5}\right)=\cdots=g\left(a_{8}\right)=0$

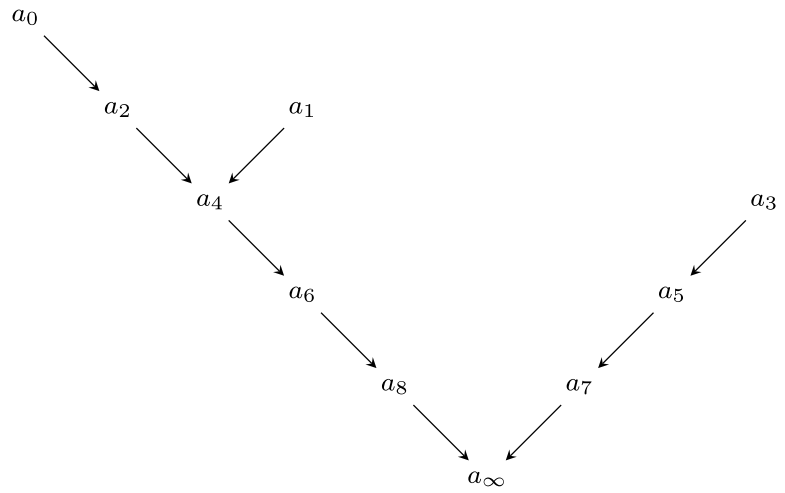

Therefore, we find that $a_{1}, a_{2}$, and $a_{3}$ are in the interval $(0,9), a_{4}, a_{5}, a_{6}, a_{7}$ are in the interval $(9,18)$, and $a_{8}$ is in the interval $(18,27)$.

Now we need to compare the remainders $r_{1}, \ldots, r_{8}$ of the 9 -generators $a_{1}, \ldots, a_{8}$. We already know that $r_{1}<r_{2}<r_{3}$, and $r_{4}<r_{5}<r_{6}<r_{7}$. Let us compare $r_{1}$ and $r_{4}$. By Lemma 14 and the tree $T_{\Delta}$, we get $N_{1,4}=2$. Therefore, by Corollary $7, K_{1,4}=1$, which means that $a_{4}-a_{1}<9$. Therefore, $r_{4}=a_{4}-9<a_{1}=r_{1}$.

Similarly, one computes that $r_{5}<r_{1}, r_{2}<r_{6}<r_{3}$, and $r_{7}>r_{3}$. Therefore, we get

$$
r_{4}<r_{5}<r_{1}<r_{2}<r_{6}<r_{3}<r_{7}
$$

To compare $r_{8}$ with the rest of the remainders, we compute

$$
N_{1,8}=N_{2,8}=4, \quad N_{3,8}=N_{4,8}=3, \quad N_{5,8}=N_{6,8}=2, \quad N_{7,8}=1,
$$

and

$$
K_{1,8}=K_{2,8}=K_{3,8}=K_{4,8}=2, \quad K_{5,8}=K_{6,8}=K_{7,8}=1 .
$$

So, $r_{8}=a_{8}-18<a_{i}-9=r_{i}$ for $i=5,6,7, r_{8}=a_{8}-18<a_{i}=r_{i}$ for $i=1,2,3$, and $r_{8}=a_{8}-18>a_{4}-9=r_{4}$. Therefore,

$$
r_{4}<r_{8}<r_{5}<r_{1}<r_{2}<r_{6}<r_{3}<r_{7}
$$

or

$$
\begin{aligned}
& r_{4}=1, \quad r_{8}=2, \quad r_{5}=3, \quad r_{1}=4, \quad r_{2}=5, \\
& r_{6}=6, \quad r_{3}=7, \quad r_{7}=8 \text {. }
\end{aligned}
$$

Finally,

$$
\begin{aligned}
& a_{0}=0, \quad a_{1}=r_{1}=4, \quad a_{2}=r_{2}=5, \quad a_{3}=r_{3}=7, \quad a_{4}=r_{4}+9=10, \\
& a_{5}=r_{5}+9=12, \quad a_{6}=r_{6}+9=15, \quad a_{7}=r_{7}+9=17,
\end{aligned}
$$

and

$$
a_{8}=r_{8}+18=20 \text {. }
$$




\subsection{Bounce path and statistic}

In the case $m=k n+1$ the path $a_{0} \rightarrow a_{b_{0}^{0}} \rightarrow a_{b_{0}^{0}+b_{1}^{0}} \rightarrow \cdots \rightarrow a_{b_{0}^{0}+\cdots+b_{s}^{0}}$ in the tree $T_{\Delta}$ can be compared with the bounce path, constructed by J. Haglund [12] in the case $m=n+1$ and generalized by N. Loehr [17] for the case $m=k n+1$. The numbers $b_{0}^{0}, \ldots, b_{s}^{0}$ are equal to the horizontal steps in the bounce path. Here we recall Loehr's definition and check that it matches with the path from $a_{0}$ to $a_{k n}$ in the tree $T_{\Delta}$. We also generalize the bounce path and statistic to the case $m=k n-1$ by considering the path from $a_{0}$ to $a_{\infty}$.

Definition 14 [17] Let $D \in Y_{m, n}$, where $m=k n+1$. The bounce path for $D$ is defined as follows. We start from the northwest corner. We alternate southward and eastward steps with the first step going southward. We always stay outside the diagram $D$.

On each southward step we go south until we hit a horizontal piece of the boundary of $D$. Each eastward step is equal to the sum of the last $k$ southward steps (if there were less than $k$ southward steps yet, then it is equal to the sum of all preceding southward steps).

Let us introduce the coordinates so that the southwest corner is $(0,0)$. Then the bounce path starts from $(0, n)$ and finishes at $(k n, 0)$. The bounce statistic is defined as follows:

Definition 15 [17] Let $\left(v_{0}, \ldots, v_{a}\right)$ and $\left(h_{0}, \ldots, h_{a}\right)$ be the vertical and horizontal steps of the bounce path, respectively. Then bounce $(D)$ is given by the formula:

$$
\operatorname{bounce}(D):=\left(n-v_{0}\right)+\left(n-v_{0}-v_{1}\right)+\cdots+\left(n-\sum_{0 \leq i \leq a} v_{i}\right) \text {. }
$$

In other words, bounce $(D)$ is equal to the sum of vertical coordinates of the southwest corners of the bounce path.

Let now $\Delta$ be a semimodule over $\Gamma_{m, n}, m=k n+1$. Let $D$ be the corresponding Young diagram, and, as in the Sect. 2.3, $G_{k n+1, n}(D)$ be the diagram with columns $g\left(a_{0}\right), \ldots, g\left(a_{k n}\right)$.

Let $T_{\Delta}$ be the bounce tree, and $a_{0} \rightarrow a_{b_{0}^{0}} \rightarrow \cdots \rightarrow a_{b_{0}^{0}+\cdots+b_{s}^{0}}=a_{k n}$ be the path from $a_{0}$ to $a_{k n}$ in it.

Theorem 17 The horizontal steps $\left(h_{0}, \ldots, h_{a}\right)$ of the bounce path for the diagram $G_{k n+1, n}(D)$ are equal to $\left(b_{0}^{0}, \ldots, b_{s}^{0}\right)$. In particular, $a=s$.

Proof Recall the formulas (4) and (5) (we plug $l=0$ ):

$$
b_{i}^{0}= \begin{cases}\sum_{j=i-k}^{i-1} c_{j}^{0} & \text { if } i \neq k-1, \\ 1+\sum_{j=i-k}^{i-1} c_{j}^{0} & \text { if } i=k-1\end{cases}
$$


and

$$
c_{i}^{0}=g\left(a_{b_{0}^{0}+b_{1}^{0}+\cdots+b_{i-1}^{0}}\right)-g\left(a_{b_{0}^{0}+b_{1}^{0}+\cdots+b_{i}^{0}}\right), \quad c_{0}^{0}=g\left(a_{0}\right)-g\left(a_{b_{0}^{0}}\right) .
$$

where $c_{i}^{0}$ is the number of $n$-cogenerators in the interval $(i n,(i+1) n]$.

We immediately see that

$$
c_{i}^{0}= \begin{cases}0 & \text { if } i<-2, \\ 1 & \text { if } i=-2, \\ n-g\left(a_{0}\right)-1 & \text { if } i=-1 .\end{cases}
$$

Indeed, $-n$ is the smallest $n$-cogenerator, and there are exactly $n-g\left(a_{0}\right) n$ cogenerators less than zero.

For $i \geq 0$ the recurrence relations on the numbers $c_{i}^{0}$ and $b_{i}^{0}$ are almost the same as the definitions of the vertical and horizontal steps of the bounce path, respectively. There are two differences:

1. The first vertical step equals $v_{0}=n-g\left(a_{0}\right)=c_{-1}^{0}+1=c_{-1}^{0}+c_{-2}^{0}$.

2. For $i=k-1$ one has $b_{k-1}^{0}=1+\sum_{j=-1}^{k-2} c_{j}^{0}=\sum_{j=-2}^{k-2} c_{j}^{0}$.

One immediately sees that those differences cancel each other. Therefore one gets

$$
v_{i}= \begin{cases}c_{i-1}^{0} & \text { if } i>0, \\ c_{-1}^{0}+c_{-2}^{0} & \text { if } i=0\end{cases}
$$

and

$$
h_{i}=b_{i}^{0} \quad \text { for all } i \geq 0 .
$$

Theorem 18 We get the following relation:

$$
\text { bounce }\left(G_{k n+1, n}(D)\right)=\delta-|D| \text {. }
$$

Proof Indeed, we have

$$
\delta-|D|=\sharp\left(\mathbb{Z}_{>0} \backslash \Delta\right)=\sum_{i=0}^{\infty} \sharp((i n,(i+1) n] \backslash \Delta) .
$$

By Lemma $10,\left[a_{b_{0}^{0}+\cdots+b_{i}^{0}}, n i\right] \subset \Delta$. We get

$$
\sharp((i n,(i+1) n] \backslash \Delta)=g\left(a_{b_{0}^{0}+\cdots+b_{i-1}^{0}}\right) \quad \text { and } \quad \sharp((0, n] \backslash \Delta)=g\left(a_{0}\right) .
$$

Therefore,

$$
\delta-|D|=g\left(a_{0}\right)+g\left(a_{b_{0}^{0}}\right)+g\left(a_{b_{0}^{0}+b_{1}^{0}}\right)+\cdots+g\left(a_{k n}\right),
$$

Note that this matches the definition of the bounce statistic. 
Fig. 11 The diagram $G_{7,3}(\Delta)$ with the bounce path for the $\Gamma_{3,7}$-semimodule $\Delta=\{0,2,3,5,6,7,8,9, \ldots\}$. Here the 7 -generators are $0,2,3,5,6,8,11$, $g(0)=g(2)=g(3)=1$, and $g(5)=g(6)=g(8)=g(11)=0$

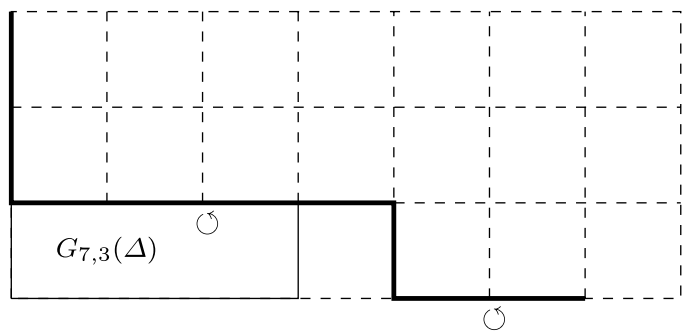

Remark 3 It follows from the proof of Theorem 18 that the number $\sharp([i n,(i+1) n] \backslash$ $\Delta$ ) is equal to the vertical coordinate of the $i$ th southwest corner of the bounce path.

We illustrate the bounce path in Fig. 11.

We use the above observations to generalize the bounce path and statistic to the case $m=k n-1$. Let $\Delta$ be a $\Gamma_{m, n}$-semimodule, $m=k n-1, D=D(\Delta)$. Consider the bounce tree $T_{\Delta}$. Let $a_{0} \rightarrow a_{b_{0}^{0}} \rightarrow \cdots \rightarrow a_{b_{0}^{0}+\cdots+b_{s}^{0}} \rightarrow a_{\infty}$ be the path from $a_{0}=0$ to $a_{\infty}$ in the tree $T_{\Delta}$. Consider the Young diagram $G_{k n-1, n}(D) \in Y_{m, n}$.

Definition 16 The bounce path for $G_{k n-1, n}(D)$ starts at the northwest corner. It consists of alternating southward and eastward steps, starting with a southward step. On each southward step we go south until we hit a horizontal piece of the boundary of $G_{k n-1, n}(D)$. The $i$ th eastward step equals $b_{i}^{0}$, starting with $i=0$.

The bounce statistic is defined in the same way as in the $m=k n+1$ case:

Definition 17 Let $\left(v_{0}, \ldots, v_{s}\right)$ and $\left(h_{0}, \ldots, h_{s}\right)$ be the vertical and horizontal steps of the bounce path, respectively. Then the bounce $\left(G_{k n-1, n}(D)\right)$ is given by

$$
\operatorname{bounce}\left(G_{k n-1, n}(D)\right)=\left(n-v_{0}\right)+\left(n-v_{0}-v_{1}\right)+\cdots+\left(n-\sum_{0 \leq i \leq s} v_{i}\right) \text {. }
$$

In other words, bounce $\left(G_{k n-1, n}(D)\right)$ is equal to the sum of vertical coordinates of the southwest corners of the bounce path.

The following formula relating the bounce of $G_{k n-1, n}(D)$ with the area of $D$ is proved in the same way as in the $m=k n+1$ case:

Theorem 19 We get the following relation:

$$
\operatorname{bounce}\left(G_{k n-1, n}(D)\right)=\delta-|D| \text {. }
$$

Finally, we expand the recurrence relations involved in the definition of the bounce path in this case to get a simple description of the bounce path in terms of the diagram $G_{k n-1, n}(D)$. This is parallel with the proof of Theorem 17 in the $m=k n+1$ case. 
Fig. 12 The diagram $G_{8,3}(\Delta)$ with the bounce path for the $\Gamma_{3,8}$-semimodule $\Delta=\{0,3,4,6,7,8,9, \ldots\}$. Here the 8-generators are

$0,3,4,6,7,9,10,13, g(0)=2$, $g(3)=g(4)=1$, and $g(6)=g(7)=g(9)=g(10)=$ $g(13)=0$

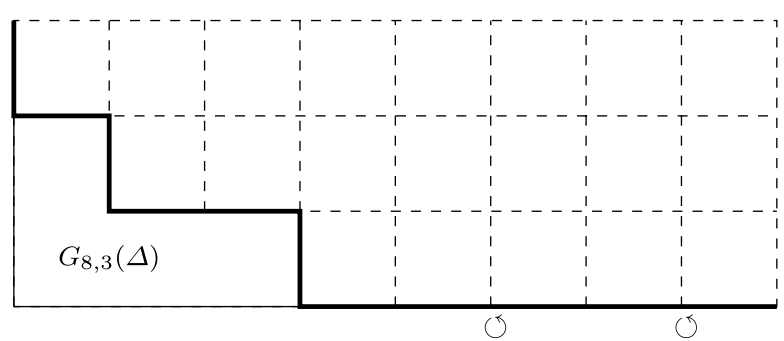

Recall the formulas (7) and (8) (we plug $l=0$ ):

$$
b_{i}^{0}= \begin{cases}\sum_{j=i-k}^{i-1} c_{j}^{0} & \text { if } i \neq k-1, \\ -1+\sum_{j=i-k}^{i-1} c_{j}^{0} & \text { if } i=k-1,\end{cases}
$$

and

$$
c_{i}^{0}=g\left(a_{b_{0}^{0}+b_{1}^{0}+\cdots+b_{i-1}^{0}}\right)-g\left(a_{b_{0}^{0}+b_{1}^{0}+\cdots+b_{i}^{0}}\right), \quad c_{0}^{0}=g\left(a_{0}\right)-g\left(a_{b_{0}^{0}}\right),
$$

where $c_{i}^{0}$ is the number of $n$-cogenerators in the interval $[i n,(i+1) n)$.

We immediately see that

$$
c_{i}^{0}= \begin{cases}0 & \text { if } i<-1, \\ n-g\left(a_{0}\right) & \text { if } i=-1 .\end{cases}
$$

Indeed, $-n$ is the smallest $n$-cogenerator, and there are exactly $n-g\left(a_{0}\right)$ $n$-cogenerators less than zero.

Comparing the recurrence relations one immediately gets

$$
v_{i}=c_{i-1}^{0} \quad \text { and } \quad h_{i}=b_{i}^{0} \quad \text { for all } i \geq 0 .
$$

Therefore, the bounce path in the $m=k n-1$ case is constructed in the same way as in the $m=k n+1$ case, except that the $(k-1)$ st horizontal step is shorter by 1 (we count steps starting from 0 ):

$$
h_{i}= \begin{cases}\sum_{j=i-k}^{i-1} v_{j} & \text { if } i \neq k-1, \\ -1+\sum_{j=i-k}^{i-1} v_{j} & \text { if } i=k-1 .\end{cases}
$$

We illustrate the bounce path in Fig. 12.

Let us return to the $(3,8)$-semimodule $\Delta=\{0,3,4,6,7,8,9, \ldots\}$ from example in Fig. 9. The 8-generators are $0,3,4,6,7,9,10,13$, so the diagram $G_{8,3}(\Delta)$ with the bounce path is shown in Fig. 12.

\section{Bijective proof of symmetry for $m \leq 3$}

In this section we give a bijective proof of the identity $c_{n, m}(q, t)=c_{n, m}(t, q)$ for $m=2$ and $m=3$. 
The $(2 k+1,2)$ case is very simple. We should consider Young diagrams in the set $Y_{2 k+1,2}$. Such a diagram $D_{i}$ can have only one row with $i \leq k$ boxes in it, and $\left|D_{i}\right|=h_{+}\left(D_{i}\right)=i$. Therefore, the polynomial $c_{2 k+1,2}(q, t)$ is given by the following formula:

$$
c_{2 k+1,2}(q, t)=q^{k}+q^{k-1} t+\cdots+q t^{k-1}+t^{k},
$$

which is obviously symmetric in $q$ and $t$. The corresponding involution on diagrams sends the diagram $D_{i}$ to $D_{k-i}$.

The case $(n, 3)$ turns out to be more subtle. We consider Young diagrams in the set $Y_{n, 3}$. Such a diagram $D_{\alpha, \beta}$ has two rows of length $\alpha$ and $\beta, \alpha \leq \beta$. Moreover,

$$
\alpha \leq k:=\left\lfloor\frac{n}{3}\right\rfloor, \quad \beta \leq\left\lfloor\frac{2 n}{3}\right\rfloor .
$$

Consider the map $\phi: Y_{n, 3} \rightarrow \mathbb{Z}^{2}, \phi(D):=\left(\delta-|D|, h_{+}(D)\right)$.

Theorem 20 The map $\phi$ is injective. The image of $\phi$ is the set of integer points inside the triangle:

$$
\mathbf{T}_{\delta}:=\{(a, b): a+b \leq \delta, a+2 b \geq \delta, 2 a+b \geq \delta\}
$$

Proof The area of $D_{\alpha, \beta}$ equals $\alpha+\beta$. To compute $h_{+}\left(D_{\alpha, \beta}\right)$, we consider three cases:

1. $0 \leq \alpha \leq \beta \leq k$. In this case one can check that $h_{+}\left(D_{\alpha, \beta}\right)=\beta$. Therefore, $\phi\left(D_{\alpha, \beta}\right)=(\delta-\alpha-\beta, \beta)$, and the image of $\phi$ is given by the formula

$$
\left\{(a, b) \in \mathbf{T}_{\delta} \cap \mathbb{Z}^{2}: b \leq k\right\} .
$$

2. $\beta>k, \beta-\alpha \leq k$. In this case $h_{+}\left(D_{\alpha, \beta}\right)=2 \beta-k, \phi\left(D_{\alpha, \beta}\right)=(\delta-\alpha-\beta, 2 \beta-k)$, and the image of $\phi$ is given by the formula

$$
\left\{(a, b) \in \mathbf{T}_{\delta} \cap \mathbb{Z}^{2}: b>k, b+k \text { even }\right\} .
$$

Indeed, the boxes contributing to $h_{+}\left(D_{\alpha, \beta}\right)$ form three groups of sizes $\alpha, \beta-\alpha$, and $\beta-k$, hence

$$
h_{+}\left(D_{\alpha, \beta}\right)=(\beta-\alpha)+\alpha+(\beta-k)=2 \beta-k .
$$

We show these boxes in Fig. 13.

3. $\beta-\alpha>k$. In this case $h_{+}\left(D_{\alpha, \beta}\right)=2 \alpha+k+1, \phi\left(D_{\alpha, \beta}\right)=(\delta-\alpha-\beta, 2 \alpha+k+1)$, and the image of $\phi$ is given by the formula

$$
\left\{(a, b) \in \mathbf{T}_{\delta} \cap \mathbb{Z}^{2}: b>k, b+k \text { odd }\right\} .
$$

Note that these three image sets cover all integer points in $\mathbf{T}_{\delta}$ with no overlaps.

Note that the triangle $\mathbf{T}_{\delta}$ is symmetric with respect to the diagonal $a=b$. Therefore, one gets the following 
Fig. 13 Boxes contributing to $h_{+}\left(D_{\alpha, \beta}\right)$ for $\beta>k, \beta-\alpha \leq k$

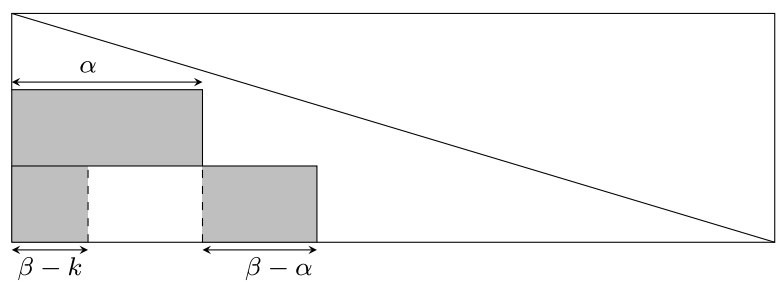

Corollary 10 There exists an involution $\mathbf{i}$ on $Y_{n, 3}$ such that

$$
\delta-|D|=h_{+}(\mathbf{i}(D)) .
$$

In particular, the polynomial $c_{n, 3}(q, t)$ is symmetric in $q$ and $t$.

Proof The involution $\mathbf{i}$ is given by the formula $\mathbf{i}=\phi^{-1} \circ \mathbf{s} \circ \phi$, where $\mathbf{s}: \mathbb{R}^{2} \rightarrow \mathbb{R}^{2}$ is the symmetry with respect to the diagonal $a=b$.

Acknowledgements The authors are grateful to D. Armstrong, L. Göttsche, J. Haglund, A. Iarrobino, L. Migliorini, A. Oblomkov, J. Rasmussen and V. Shende for useful discussions. Special thanks to K. Lee, L. Li and N. Loehr for communicating us their proof of Conjecture 1 for $m \leq 4$. The research of E.G. was partially supported by the grants RFBR-10-01-00678, NSh-8462.2010.1 and the Simons foundation.

\section{References}

1. Altman, A., Iarrobino, A., Kleiman, S.: Irreducibility of the compactified Jacobian. In: Nordic Summer School NAVF, Oslo, 1976. Noordhoff, Groningen (1977)

2. Anderson, J.: Partitions which are simultaneously $t_{1}$ - and $t_{2}$-core. Discrete Math. 248(1-3), 237-243 (2002)

3. Armstrong, D.: Rational Catalan Combinatorics, slides from a talk at JMM 2012, Boston

4. Beauville, A.: Counting rational curves on K3-surfaces. Duke Math. J. 97, 99-108 (1999)

5. Delgado de la Mata, F.: Gorenstein curves and symmetry of the semigroup of values. Manuscr. Math. 61(3), 285-296 (1988)

6. Ford, B., Mai, H., Sze, L.: Self-conjugate $p$ - and $q$-core partitions and blocks of $A_{n}$. J. Number Theory 129, 858-865 (2009)

7. Garsia, A., Haiman, M.: A remarkable $q, t$-Catalan sequence and $q$-Lagrange inversion. J. Algebr. Comb. 5(3), 191-244 (1996)

8. Garsia, A., Haglund, J.: A proof of the $q$, $t$-Catalan positivity conjecture. Discrete Math. 256(3), 677717 (2002)

9. Gorsky, E., Mazin, M.: Compactified Jacobians and $q, t$-Catalan numbers. J. Comb. Theory, Ser. A 120, 49-63 (2013)

10. Gorsky, E., Oblomkov, A., Rasmussen, J., Shende, V.: Torus knots and the rational DAHA. arXiv: 1207.4523

11. Greuel, G.-M., Pfister, G.: Moduli spaces for torsion free modules on curve singularities I. J. Algebr. Geom. 2, 81-135 (1993)

12. Haglund, J.: Conjectured statistics for the $q$, $t$-Catalan numbers. Adv. Math. 175(2), 319-334 (2003)

13. Haglund, J.: The $q, t$-Catalan Numbers and the Space of Diagonal Harmonics. With an Appendix on the Combinatorics of Macdonald Polynomials. University Lecture Series, vol. 41. American Mathematical Society, Providence (2008)

14. Hikita, T.: Affine Springer fibers of type $A$ and combinatorics of diagonal coinvariants. arXiv: 1203.5878

15. Kunz, E.: The value-semigroup of a one-dimensional Gorenstein ring. Proc. Am. Math. Soc. 25, 748$751(1970)$ 
16. Lee, K., Li, L., Loehr, N.: Combinatorics of certain higher $q$, $t$-Catalan polynomials: chains, joint symmetry, and the Garsia-Haiman formula. arXiv:1211.2191

17. Loehr, N.: Conjectured statistics for the higher $q, t$-Catalan sequences. Electron. J. Comb. 12, 9 (2005)

18. Loehr, N., Warrington, G.: A continuous family of partition statistics equidistributed with length. J. Comb. Theory, Ser. A 116, 379-403 (2009)

19. Lusztig, G., Smelt, J.M.: Fixed point varieties on the space of lattices. Bull. Lond. Math. Soc. 23(3), 213-218 (1991)

20. Milnor, J.W., Stasheff, J.D.: Characteristic Classes. Annals of Mathematics Studies, vol. 76. Princeton University Press, Princeton (1974)

21. Oblomkov, A., Shende, V.: The Hilbert scheme of a plane curve singularity and the HOMFLY polynomial of its link. Duke Math. J. 161(7), 1277-1303 (2012)

22. Oblomkov, A., Rasmussen, J., Shende, V.: The Hilbert scheme of a plane curve singularity and the HOMFLY homology of its link. arXiv: 1201.2115

23. Piontkowski, J.: Topology of the compactified Jacobians of singular curves. Math. Z. 255(1), 195-226 (2007)

24. Puchta, J.C.: Partitions which are $p$ - and $q$-core. Integers 1, A6 (2001). 3 pp. 